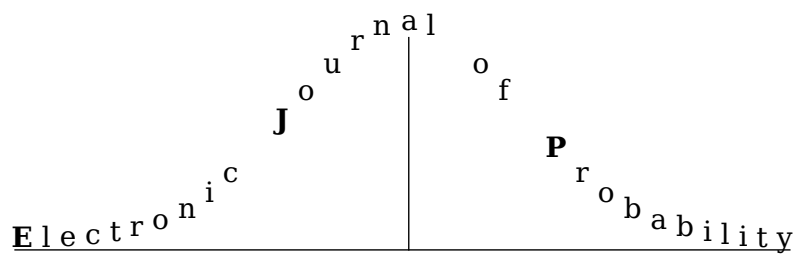

Electron. J. Probab. 25 (2020), article no. 85, 1-31.

ISSN: 1083-6489 https://doi.org/10.1214/20-EJP489

\title{
Coalescence estimates for the corner growth model with exponential weights*
}

\author{
Timo Seppäläinen $^{\dagger} \quad$ Xiao Shen ${ }^{\ddagger}$
}

\begin{abstract}
We establish estimates for the coalescence time of semi-infinite directed geodesics in the planar corner growth model with i.i.d. exponential weights. There are four estimates: upper and lower bounds on the probabilities of both fast and slow coalescence on the correct spatial scale with exponent $3 / 2$. Our proofs utilize a geodesic duality introduced by Pimentel and properties of the increment-stationary last-passage percolation process. For fast coalescence our bounds are new and they have matching optimal exponential order of magnitude. For slow coalescence we reproduce bounds proved earlier with integrable probability inputs, except that our upper bound misses the optimal order by a logarithmic factor.
\end{abstract}

Keywords: coalescence exit time; fluctuation exponent; geodesic; last-passage percolation; Kardar-Parisi-Zhang; random growth model.

AMS MSC 2010: 60K35; 60K37.

Submitted to EJP on November 27, 2019, final version accepted on June 27, 2020.

\section{Introduction}

Random growth models of the first- and last-passage type have been a central part of the mathematical theory of spatial stochastic processes since the seminal work of Eden [13] and Hammersley and Welsh [18]. In these models, growth proceeds along optimal paths called geodesics, determined by a random environment. The interesting and challenging objects of study are the directed semi-infinite geodesics. These pose an immediate existence question because they are asymptotic objects and hence cannot be defined locally in a simple manner. Once the existence question is resolved, questions concerning their multiplicity and geometric behavior such as coalescence arise.

Techniques for establishing the existence, uniqueness, and coalescence of semiinfinite geodesics were first introduced by Newman and co-authors in the 1990s [19,

\footnotetext{
*T. Seppäläinen was partially supported by National Science Foundation grants DMS-1602486 and DMS1854619, and by the Wisconsin Alumni Research Foundation.

${ }^{\dagger}$ University of Wisconsin-Madison. E-mail: seppalai@math.wisc.edu

${ }^{\ddagger}$ University of Wisconsin-Madison. E-mail: xshen@math.wisc.edu
} 
$20,22,23]$ in the context of planar undirected first-passage percolation (FPP) with i.i.d. weights. These methods were subsequently applied to the exactly solvable planar directed last-passage percolation (LPP) model with i.i.d. exponential weights by Ferrari and Pimentel [16] and Coupier [12]. This model is also known as the exponential corner growth model (CGM).

A key technical point here is that the strict curvature hypotheses of Newman's work can be verified in the exactly solvable LPP model. A second key feature is that the exponential LPP model can be coupled with the totally asymmetric simple exclusion process (TASEP). This connection provides another suite of powerful tools for analyzing exponential LPP.

The work of [12] and [16] established for the exponential LPP model that, almost surely for a fixed direction, directed semi-infinite geodesics from each lattice point are unique and they coalesce. An alternative approach to these results was recently developed by one of the authors [28], by utilizing properties of the increment-stationary LPP process.

Once coalescence is known, attention turns to quantifying it: how fast do semi-infinite geodesics started from two distinct points coalesce? The scaling properties of planar models in the Kardar-Parisi-Zhang (KPZ) class come into the picture here. This class consists of interacting particle systems, random growth models and directed polymer models in two dimensions (one of which can be time) that share universal fluctuation exponents and limit distributions from random matrix theory. For surveys of the field, see $[11,25]$.

It is expected that, subject to mild moment assumptions on the weights, planar FPP and LPP are members of the KPZ class. It is conjectured in general and proved in exactly solvable cases that a geodesic of length $N$ fluctuates on the scale $N^{2 / 3}$. Thus if two semi-infinite geodesics start at distance $k$ apart, we expect coalescence to happen on the scale $k^{3 / 2}$.

The first step in the study of the coalescence exponent was taken by Wüthrich [29]. He proved a lower bound with exponent $3 / 2-\epsilon$ for LPP on planar Poisson points. This was the first application of the first-passage percolation techniques of Newman and coauthors in the context of an exactly solvable last-passage percolation model. The second step in this direction was taken by Pimentel [24] for the exponential CGM. By relying on the TASEP connection, he proved that in a fixed direction, the so-called dual geodesic graph is equal in distribution (modulo a lattice reflection) to the original geodesic tree. Next, by appeal to fluctuation bounds derived by coupling techniques in [4], he derived an asymptotic lower bound on the coalescence time, with the expected exponent $3 / 2$.

The next step taken by Basu, Sarkar, and Sly [7] utilized the considerably more powerful estimates from integrable probability. For the upper bound on the coalescence time, they established not only the correct order of magnitude $k^{3 / 2}$ but also upper and lower probability bounds of matching orders of magnitude. In the same paper the original estimate of Pimentel was also improved significantly.

Our goal in taking up the speed of coalescence is the development of proof techniques that rely only on the stationary version of the model and avoid both the TASEP connection and integrable probability. The applicability of this approach then covers all $1+1$ dimensional KPZ models with a tractable stationary version. This includes not only various last-passage models in both discrete and continuous space, but also the four currently known solvable positive temperature polymer models [10]. Extension beyond solvable models may also be possible, as indicated by the exact KPZ fluctuation exponents derived in [5] for a class of zero-range processes outside currently known exactly solvable models. This is work left for the future. Another somewhat philosophical point is that 
capturing exponents should be possible without integrable probability. This has been demonstrated for fluctuation exponents by [4] for the exponential LPP and by [26] for a positive-temperature directed polymer model.

The results of this paper come from a unified approach based on controlling the exit point of the geodesic in a stationary LPP process and on Pimentel's duality of geodesics and dual geodesics. This involves coupling, random walk estimates, planar monotonicity, and distributional properties of the stationary LPP process. Here are the precise contributions of the present paper (details in Section 2.2):

(i) The upper and lower bounds for slow coalescence originally due to Basu et al. [7], though our upper bound falls short of the optimal order by a logarithmic factor (Theorem 2.2). Our contribution here is to give a proof without integrable probability inputs.

(ii) Upper and lower bounds for fast coalescence of matching exponential order (Theorem 2.3). These are new results.

(iii) A lower bound on the transversal fluctuations of a directed semi-infinite geodesic which improves bounds obtainable without integrable probability (Theorem 2.8).

(iv) Strengthened exit time estimates for the stationary LPP process without integrable probability, some uniform over endpoints beyond a given distance (Theorems $4.1,4.4,4.5)$.

We mention two more general but related points about the exponential CGM.

(a) When all directions are considered simultaneously, the overall picture of semiinfinite geodesics is richer than the simple almost-sure-uniqueness-plus-coalescence valid for a fixed direction. Part of this was already explained by Coupier [12]. Recently the global picture of uniqueness and coalescence was captured in [21]. Coalescence bounds that go beyond the almost surely unique geodesics in a fixed direction are left as an open problem for the future.

(b) Various geometric features of the exponential LPP process can now be proved without appeal to properties of TASEP. An exception is a deep result of Coupier [12] on the absence of triple geodesics in any random direction. This fact currently has no proof except the original one that relies on the TASEP speed process introduced in [1].

\section{Organization of the paper}

Precise definition of the exponential LPP model and the main results appear in Section 2. Section 3 collects known facts about the CGM used in the proofs. This includes properties of the stationary growth process and the construction of the directed semi-infinite geodesics in terms of Busemann functions. Section 4 derives new exit time estimates for the geodesic of the stationary growth process, stated as Theorems 4.1, 4.4, and 4.5. In the final Section 5 the exit time estimates and duality are combined to prove the main results of Section 2. The appendix contains a random walk estimate and a moment bound on the Radon-Nikodym derivative between two product-form exponential distributions.

\section{Notation and conventions}

Points $x=\left(x_{1}, x_{2}\right), y=\left(y_{1}, y_{2}\right) \in \mathbb{R}^{2}$ are ordered coordinatewise: $x \leq y$ iff $x_{1} \leq y_{1}$ and $x_{2} \leq y_{2}$. The $\ell^{1}$ norm is $|x|_{1}=\left|x_{1}\right|+\left|x_{2}\right|$. The origin of $\mathbb{R}^{2}$ is denoted by both 0 and $(0,0)$. The two standard basis vectors are $e_{1}=(1,0)$ and $e_{2}=(0,1)$. For $a \leq b$ in $\mathbb{Z}^{2}$, $\llbracket a, b \rrbracket=\left\{x \in \mathbb{Z}^{2}: a \leq x \leq b\right\}$ is the rectangle in $\mathbb{Z}^{2}$ with corners $a$ and $b . \llbracket a, b \rrbracket$ is a segment if $a$ and $b$ are on the same horizontal or vertical line. We use $\llbracket a-e_{1}, a \rrbracket, \llbracket a-e_{2}, a \rrbracket$ 


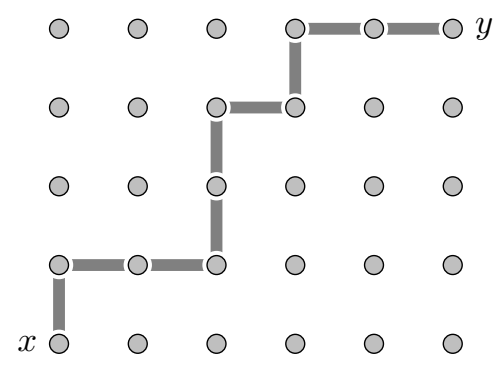

Figure 2.1: An up-right path between two integer points $x$ and $y$.

to denote unit edges when it is clear from the context. Subscripts indicate restricted subsets of the reals and integers: for example $\mathbb{Z}_{>0}=\{1,2,3, \ldots\}$ and $\mathbb{Z}_{>0}^{2}=\left(\mathbb{Z}_{>0}\right)^{2}$ is the positive first quadrant of the planar integer lattice. For $0<\alpha<\infty, X \sim \operatorname{Exp}(\alpha)$ means that the random variable $X$ has exponential distribution with rate $\alpha$, in other words $P(X>t)=e^{-\alpha t}$ for $t>0$ and $E(X)=\alpha^{-1}$.

\section{Main results}

\subsection{The corner growth model and semi-infinite geodesics}

The standard exponential corner growth model (CGM) is defined on the planar integer lattice $\mathbb{Z}^{2}$ through independent and identically distributed (i.i.d.) weights $\left\{\omega_{z}\right\}_{z \in \mathbb{Z}^{2}}$, indexed by the vertices of $\mathbb{Z}^{2}$, with marginal distribution $\omega_{z} \sim \operatorname{Exp}(1)$. The last-passage value $G_{x, y}$ between two coordinatewise-ordered vertices $x \leq y$ of $\mathbb{Z}^{2}$ is the maximal total weight of an up-right nearest-neighbor path from $x$ to $y$ :

$$
G_{x, y}=\max _{z \cdot \in \Pi^{x, y}} \sum_{k=0}^{|y-x|_{1}} \omega_{z_{k}}
$$

where $\Pi^{x, y}$ is the set of paths $z_{\bullet}=\left(z_{k}\right)_{k=0}^{|y-x|_{1}}$ that satisfy $z_{0}=x, z_{|y-x|_{1}}=y$, and $z_{k+1}-z_{k} \in\left\{e_{1}, e_{2}\right\}$. The almost surely unique maximizing path is the point-to-point geodesic. $G_{x, y}$ is also called (directed) last-passage percolation (LPP). If $x \leq y$ fails our convention is $G_{x, y}=-\infty$.

A semi-infinite up-right path $\left(z_{i}\right)_{i=0}^{\infty}$ is a semi-infinite geodesic if it is the maximizing path between any two points on this path, that is,

$$
\forall k<l \text { in } \mathbb{Z}_{\geq 0}:\left(z_{i}\right)_{i=k}^{l} \in \Pi^{z_{k}, z_{l}} \quad \text { and } \quad G_{z_{k}, z_{l}}=\sum_{i=k}^{l} \omega_{z_{i}} .
$$

For a point $\xi \in \mathbb{R}_{\geq 0}^{2} \backslash\{0\}$, the semi-infinite path $\left(z_{i}\right)_{i=0}^{\infty}$ is $\xi$-directed if $z_{i} /\left|z_{i}\right|_{1} \rightarrow \xi /|\xi|_{1}$ as $i \rightarrow \infty$.

In the exponential CGM it is natural to index spatial directions $\xi$ by a real parameter $\rho \in(0,1)$ through the equation

$$
\xi[\rho]=\left((1-\rho)^{2}, \rho^{2}\right) .
$$

We call $\xi[\rho]$ the characteristic direction associated to parameter $\rho$. This notion acquires meaning when we discuss the stationary LPP process in Section 3. Throughout, $N$ will be a scaling parameter that goes to infinity. When $\rho$ is understood, we write

$$
v_{N}=\left(\left\lfloor N(1-\rho)^{2}\right\rfloor,\left\lfloor N \rho^{2}\right\rfloor\right)
$$



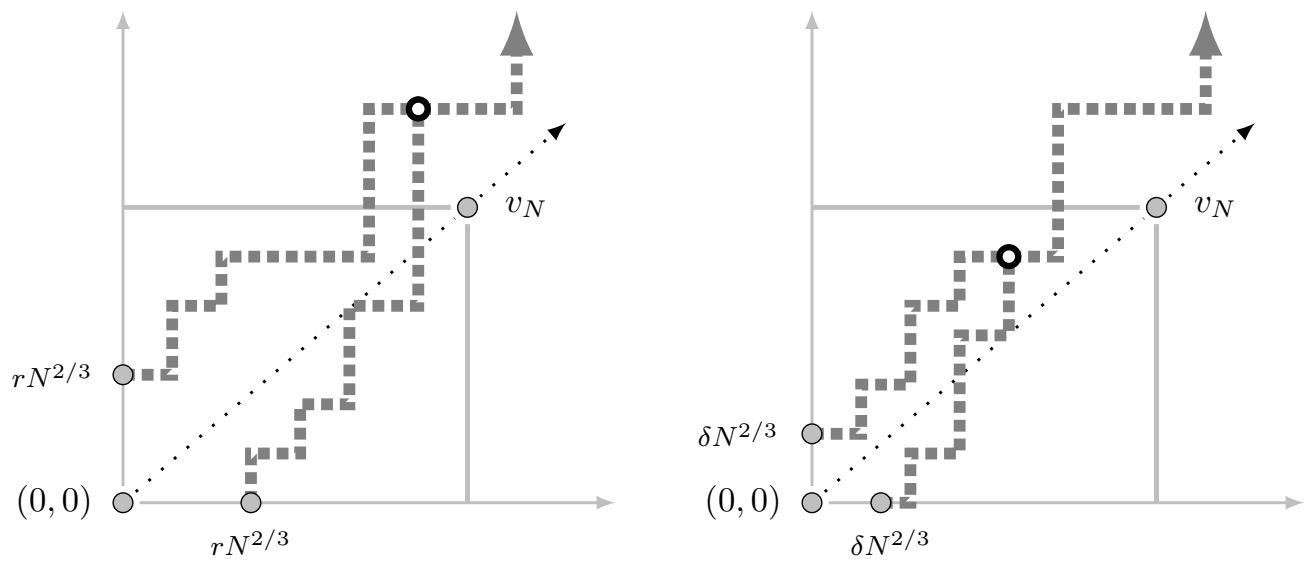

Figure 2.2: Coalescence of $\xi[\rho]$-directed semi-infinite geodesics. The black circle marks the coalescence point: on the left it is $\mathbf{z}^{\rho}\left(\left\lfloor r N^{2 / 3}\right\rfloor e_{1},\left\lfloor r N^{2 / 3}\right\rfloor e_{2}\right)$, and on the right $\mathbf{z}^{\rho}\left(\left\lfloor\delta N^{2 / 3}\right\rfloor e_{1},\left\lfloor\delta N^{2 / 3}\right\rfloor e_{2}\right)$. On the left for large $r$ the geodesics are likely to coalesce outside the rectangle $\llbracket 0, v_{N} \rrbracket$, while on the right for small $\delta$ the geodesics are likely to coalesce inside the rectangle $\llbracket 0, v_{N} \rrbracket$.

for the lattice point moving in direction $\xi[\rho]$.

The theorem below summarizes the key facts about directed semi-infinite geodesics that set the stage for our paper. It goes back to the work of Ferrari and Pimentel [16] and Coupier [12] on the CGM, and the general geodesic techniques introduced by Newman and coworkers [19, 20, 22, 23]. A different proof is given in [28].

Theorem 2.1. Fix $\rho \in(0,1)$. Then the following holds almost surely. For each $x \in \mathbb{Z}^{2}$ there is a unique $\xi[\rho]$-directed semi-infinite geodesic $\mathbf{b}^{\rho, x}=\left(\mathbf{b}_{i}^{\rho, x}\right)_{i=0}^{\infty}$ such that $\mathbf{b}_{0}^{\rho, x}=x$. For each pair $x, y \in \mathbb{Z}^{2}$, the geodesics coalesce: there is a coalescence point $\mathbf{z}^{\rho}(x, y)$ such that $\mathbf{b}^{\rho, x} \cap \mathbf{b}^{\rho, y}=\mathbf{b}^{\rho, z}$ for $z=\mathbf{z}^{\rho}(x, y)$.

\subsection{Coalescence estimates for semi-infinite geodesics in a fixed direction}

The two main results below give upper and lower bounds on the probability that two $\xi[\rho]$-directed semi-infinite geodesics initially separated by a distance of order $N^{2 / 3}$ coalesce inside the rectangle $\llbracket 0, v_{N} \rrbracket$. The theorems are separated according to whether the starting points of the geodesics are close to each other or far apart on the scale $N^{2 / 3}$. See the illustration in Figure 2.2. As introduced in Theorem $2.1, \mathbf{z}^{\rho}(x, y)$ is the coalescence point of the geodesics $\mathbf{b}^{\rho, x}$ and $\mathbf{b}^{\rho, y}$.

Theorem 2.2. For each $0<\rho<1$ there exist finite positive constants $\delta_{0}, C_{1}, C_{2}$ and $N_{0}$ that depend only on $\rho$ and for which the following holds: whenever $N \geq N_{0}$ and $N^{-2 / 3} \leq \delta \leq \delta_{0}$,

$$
C_{1} \delta \leq \mathbb{P}\left\{\mathbf{z}^{\rho}\left(\left\lfloor\delta N^{2 / 3}\right\rfloor e_{1},\left\lfloor\delta N^{2 / 3}\right\rfloor e_{2}\right) \notin \llbracket 0, v_{N} \rrbracket\right\} \leq C_{2}|\log \delta|^{2 / 3} \delta
$$

The requirement $\delta \geq N^{-2 / 3}$ in Theorem 2.2 is needed only for the lower bound and only to ensure that $\left\lfloor\delta N^{2 / 3}\right\rfloor \neq 0$.

Theorem 2.3. For each $0<\rho<1$ there exist finite positive constants $r_{0}, C_{1}, C_{2}$ and $N_{0}$ that depend only on $\rho$ and for which the following holds: whenever $N \geq N_{0}$ and 
$r_{0} \leq r \leq\left((1-\rho)^{2} \wedge \rho^{2}\right) N^{1 / 3}$,

$$
e^{-C_{1} r^{3}} \leq \mathbb{P}\left\{\mathbf{z}^{\rho}\left(\left\lfloor r N^{2 / 3}\right\rfloor e_{1},\left\lfloor r N^{2 / 3}\right\rfloor e_{2}\right) \in \llbracket 0, v_{N} \rrbracket\right\} \leq e^{-C_{2} r^{3}} .
$$

The requirement $r \leq\left((1-\rho)^{2} \wedge \rho^{2}\right) N^{1 / 3}$ in Theorem 2.3 is needed only for the lower bound and only to ensure that both geodesics start inside the rectangle $\llbracket 0, v_{N} \rrbracket$.

If we replace one of the starting points with the origin 0 , the upper bound of Theorem 2.2 and the lower bound of Theorem 2.3 hold automatically because $\mathbf{b}^{\rho, 0}$ stays between $\mathbf{b}^{\rho,\left(\left\lfloor r N^{2 / 3}\right\rfloor, 0\right)}$ and $\mathbf{b}^{\rho,\left(0,\left\lfloor r N^{2 / 3}\right\rfloor\right)}$. The following corollary states that the other two tail estimates also hold with possibly different constants under this alteration in the geometry.

Corollary 2.4. For each $0<\rho<1$ there exist finite positive constants $\delta_{0}, r_{0}, C_{1}, C_{2}$ and $N_{0}$ that depend only on $\rho$ and for which the following holds: whenever $N \geq N_{0}$, $N^{-2 / 3} \leq \delta \leq \delta_{0}$, and $r \geq r_{0}$,

(i) $\mathbb{P}\left\{\mathbf{z}^{\rho}\left(0,\left\lfloor\delta N^{2 / 3}\right\rfloor e_{1}\right) \notin \llbracket 0, v_{N} \rrbracket\right\} \geq C_{1} \delta$ and

(ii) $\mathbb{P}\left\{\mathbf{z}^{\rho}\left(0,\left\lfloor r N^{2 / 3}\right\rfloor e_{1}\right) \in \llbracket 0, v_{N} \rrbracket\right\} \leq e^{-C_{2} r^{3}}$.

Remark 2.5. Two comments about the results.

(a) The statements of the theorems are valid for $v_{N}=(\lfloor N a\rfloor,\lfloor N b\rfloor)$ for any fixed $a, b>0$, with new constants that depend also on $a, b$. The characteristic point $v_{N}$ of (2.3) is simply one natural choice.

(b) The constants in the theorems that depend on $\rho \in(0,1)$ can be taken fixed uniformly for all $\rho$ in any compact subset of $(0,1)$.

For direct comparison with [7], we state two corollaries for geodesics whose locations are not expressed in terms of the large parameter $N$.

Corollary 2.6. For each $0<\rho<1$ there exist finite positive constants $R_{0}, C_{1}$ and $C_{2}$ that depend only on $\rho$ and for which the following holds: whenever $k \geq 1$ and $R \geq R_{0}$,

$$
C_{1} R^{-2 / 3} \leq \mathbb{P}\left\{\mathbf{z}^{\rho}\left(\left\lfloor k^{2 / 3}\right\rfloor e_{1},\left\lfloor k^{2 / 3}\right\rfloor e_{2}\right) \notin \llbracket 0, v_{R k} \rrbracket\right\} \leq C_{2}(\log R)^{2 / 3} R^{-2 / 3} .
$$

Corollary 2.6 is derived from Theorem 2.2 as follows. Set $R_{0}=N_{0} \vee \delta_{0}^{-3 / 2}$. Given $k \geq 1$ and $R \geq R_{0}$, let $N=R k \geq N_{0}$ and $\delta=R^{-2 / 3} \leq \delta_{0}$. Now $k^{2 / 3}=\delta N^{2 / 3}$. The next Corollary 2.7 below is derived from Theorem 2.3 in a similar way.

Corollary 2.7. For each $0<\rho<1$ there exist finite positive constants $R_{1}, C_{1}$ and $C_{2}$ that depend only on $\rho$ and for which the following holds: whenever $k \geq 1$ and $\left((1-\rho)^{2} \wedge \rho^{2}\right)^{-1} k^{-1 / 3} \leq R \leq R_{1}$,

$$
e^{-C_{1} R^{-2}} \leq \mathbb{P}\left\{\mathbf{z}^{\rho}\left(\left\lfloor k^{2 / 3}\right\rfloor e_{1},\left\lfloor k^{2 / 3}\right\rfloor e_{2}\right) \in \llbracket 0, v_{R k} \rrbracket\right\} \leq e^{-C_{2} R^{-2}} .
$$

Again, the lower bound $R \geq\left((1-\rho)^{2} \wedge \rho^{2}\right)^{-1} k^{-1 / 3}$ is imposed only to ensure that both geodesics start inside the rectangle $\llbracket 0, v_{R k} \rrbracket$, for otherwise the probability in Corollary 2.7 is zero.

The lower bounds in Theorem 2.2 and Corollary 2.6 are optimal, but the upper bounds are not due to the logarithmic factor. Optimal upper and lower bounds (both of order $R^{-2 / 3}$ ) were proved for Corollary 2.6 by Basu, Sarkar, and Sly [7] with inputs from integrable probability. Thus in Theorem 2.2 and Corollary 2.6 our contribution is to provide bounds without relying on integrable probability.

Both upper and lower bounds in Theorem 2.3 are new. The upper bound $e^{-C_{2} r^{3}}$ of Theorem 2.3 improves significantly Pimentel's [24] asymptotic $(N \rightarrow \infty)$ upper bound $\mathrm{Cr}^{-3}$. The improved bound comes from duality and an exit time estimate with the 
optimal exponential order, obtained recently by Emrah, Janjigian, and one of the authors in [14] without integrable probability inputs. This exit time estimate was also derived independently by Bhatia [8] with integrable probability inputs. In the intervening period between Pimentel's work and the present paper, Pimentel's bound was improved to $e^{-C r^{3 / 2}}$ (without sending $N$ to infinity) in [7] with inputs from integrable probability, see [7, Remark 6.5].

It is by now well-known that over distances of order $N$, geodesics fluctuate on the scale $N^{2 / 3}$. A by-product of our proof is the following lower bound on the size of the transversal fluctuation of a semi-infinite geodesic. It is an improvement over previous bounds obtained without integrable probability (see Theorem 5.3(b) in [27]).

Theorem 2.8. For each $0<\rho<1$ there exist positive constants $C, N_{0}$ and $\delta_{0}$ that depend only on $\rho$ for which the following holds: whenever $N \geq N_{0}$ and $0<\delta \leq \delta_{0}$,

$$
\mathbb{P}\left\{\mathbf{b}^{\rho,(0,0)} \text { enters the rectangle } \llbracket v_{N}-\delta N^{2 / 3}\left(e_{1}+e_{2}\right), v_{N} \rrbracket\right\} \leq C|\log \delta|^{2 / 3} \delta .
$$

The proofs in Section 5 show that the probability in (2.8) is essentially bounded above by the probability in (2.4). With inputs from integrable probability, the upper bound $|\log \delta|^{2 / 3} \delta$ in (2.8) can be improved to $\delta$, the optimal upper bound for (2.4) obtained in [7].

We turn to develop the groundwork for the proofs.

\section{Preliminaries on the corner growth model}

This section covers aspects of the CGM used in the proofs. We provide illustrations, some intuitive arguments, and references to precise proofs. The two main results are a fluctuation upper bound for the exit point of a stationary LPP process (Theorem 3.5) and the construction of semi-infinite geodesics with Busemann functions (Theorem 3.7). These are proved in article [14] and lecture notes [27], without using anything beyond the stationary LPP process.

\subsection{Nonrandom properties}

We begin with two basic features of LPP that involve increments. We state them for our exponential case but in fact these properties do not need any probability. Let $G_{x, \text {, be }}$ defined by (2.1) and define increment variables for $a \geq x+e_{1}$ and $b \geq x+e_{2}$ by

$$
I_{a}^{x}=G_{x, a}-G_{x, a-e_{1}} \quad \text { and } \quad J_{b}^{x}=G_{x, b}-G_{x, b-e_{2}} .
$$

The first property is a monotonicity valid for planar LPP. Proof can be found for example in Lemma 4.6 of [27].

Lemma 3.1. For $y$ such that the increments are well-defined,

$$
I_{y}^{x-e_{1}} \leq I_{y}^{x} \leq I_{y}^{x-e_{2}} \text { and } J_{y}^{x-e_{2}} \leq J_{y}^{x} \leq J_{y}^{x-e_{1}} .
$$

Fix distinct lattice points $x \leq z$ and define a second LPP process $G_{z, \bullet}^{(x)}$ with base point at $z$ that uses boundary weights given by the increments of $G_{x, .}$, as illustrated in Figure 3.1. Precisely, for $y \geq z$,

$$
G_{z, y}^{(x)}=\max _{z \cdot \in \Pi^{z, y}} \sum_{k=0}^{|y-z|_{1}} \eta_{z_{k}}
$$

where the weights are given by

$$
\begin{aligned}
\eta_{z} & =0, \quad \eta_{a}=\omega_{a} \quad \text { for } a \in z+\mathbb{Z}_{>0}^{2} \text { (bulk), } \\
\eta_{z+k e_{1}} & =I_{z+k e_{1}}^{x}, \quad \eta_{z+k e_{2}}=J_{z+k e_{1}}^{x} \quad \text { for } k \geq 1 \text { (boundary). }
\end{aligned}
$$




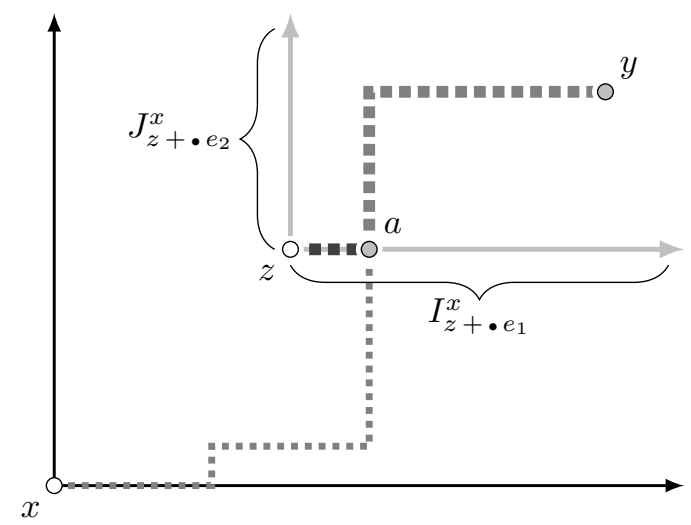

Figure 3.1: Illustration of Lemma 3.2. LPP process $G_{z, \bullet}^{(x)}$ uses boundary weights defined by the LPP process $G_{x, \bullet}$. Path $x-a-y$ is the geodesic of $G_{x, y}$ and path $z-a-y$ the geodesic of $G_{z, y}^{(x)}$. These geodesics share the segment $a-y$.

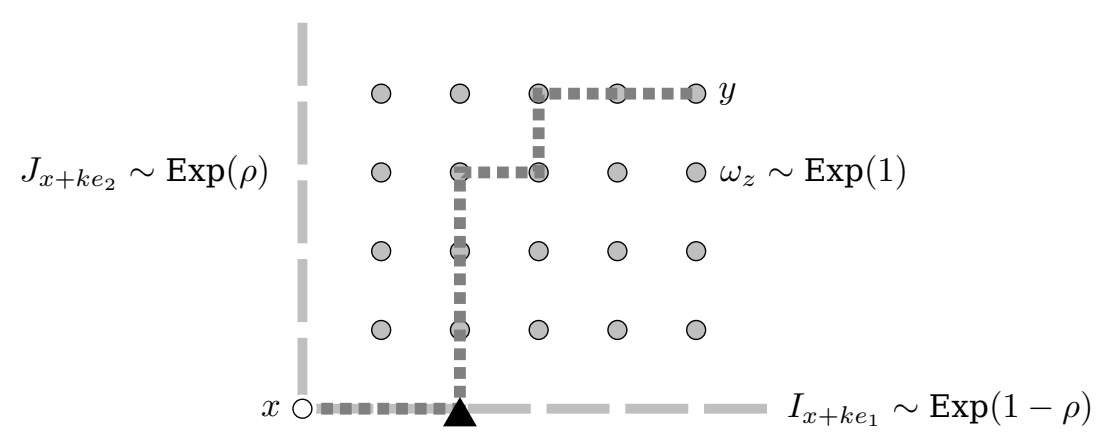

Figure 3.2: Increment-stationary LPP with base point $x$. If the dotted line were the geodesic of $G_{x, y}^{\rho}$, then the black triangle highlights the exit point, and the exit time is $\mathbf{Z}^{x \rightarrow y}=2$.

Proof of the lemma below is elementary and can be found in Lemma A.1 of [27].

Lemma 3.2. Let $x \leq z$ and $y \in z+\mathbb{Z}_{>0}^{2}$. Then the unique geodesics of $G_{x, y}$ and $G_{z, y}^{(x)}$ coincide in the quadrant $z+\mathbb{Z}_{>0}^{2}$.

\subsection{Stationary last-passage percolation}

The stationary LPP process $G^{\rho}$ is defined on a positive quadrant $x+\mathbb{Z}_{\geq 0}^{2}$ with a fixed base point $x \in \mathbb{Z}^{2}$. It is parametrized by $\rho \in(0,1)$. Start with mutually independent bulk weights $\left\{\omega_{z}: z \in x+\mathbb{Z}_{>0}^{2}\right\}$ and boundary weights $\left\{I_{x+k e_{1}}, J_{x+l e_{2}}: k, l \in \mathbb{Z}_{>0}\right\}$ with marginal distributions

$$
\omega_{z} \sim \operatorname{Exp}(1), \quad I_{x+k e_{1}} \sim \operatorname{Exp}(1-\rho), \quad \text { and } \quad J_{x+l e_{2}} \sim \operatorname{Exp}(\rho) .
$$

The probability distribution of these weights is denoted by $\mathbb{P}^{\rho}$. The LPP process $G_{x}^{\rho}$, is defined on the boundary of the quadrant by $G_{x, x}^{\rho}=0, G_{x, x+k e_{1}}^{\rho}=\sum_{i=1}^{k} I_{x+i e_{1}}$ and $G_{x, x+l e_{2}}^{\rho}=\sum_{j=1}^{l} J_{x+j e_{2}}$ for $k, l \geq 1$. In the bulk we perform LPP that uses both the 
boundary and the bulk weights: for $y=x+(m, n) \in x+\mathbb{Z}_{>0}^{2}$,

$$
G_{x, y}^{\rho}=\max _{1 \leq k \leq m}\left\{\left(\sum_{i=1}^{k} I_{x+i e_{1}}\right)+G_{x+k e_{1}+e_{2}, y}\right\} \bigvee \max _{1 \leq l \leq n}\left\{\left(\sum_{j=1}^{l} J_{x+j e_{2}}\right)+G_{x+l e_{2}+e_{1}, y}\right\} .
$$

The LPP value $G_{a, b}$ inside the braces is the standard one defined by (2.1) with the i.i.d. bulk weights $\omega$. Call the almost surely unique maximizing path a $\rho$-geodesic. The exit time $\mathbf{Z}^{x \rightarrow y}$ is the $\mathbb{Z} \backslash\{0\}$-valued random variable that records where the $\rho$-geodesic from $x$ to $y$ exits the boundary, relative to the base point $x$, with a sign that indicates choice between the axes:

$$
G_{x, y}^{\rho}= \begin{cases}\sum_{i=1}^{k} I_{x+i e_{1}}+G_{x+k e_{1}+e_{2}, y}, & \text { if } \mathbf{Z}^{x \rightarrow y}=k>0 \\ \sum_{j=1}^{l} J_{x+j e_{2}}+G_{x+l e_{2}+e_{1}, y}, & \text { if } \mathbf{Z}^{x \rightarrow y}=-l<0 .\end{cases}
$$

See Figure 3.2 for an illustration.

Define horizontal and vertical increments of $G_{x, \bullet}^{\rho}$ as

$$
I_{a}^{x}=G_{x, a}^{\rho}-G_{x, a-e_{1}}^{\rho} \quad \text { and } \quad J_{b}^{x}=G_{x, b}^{\rho}-G_{x, b-e_{2}}^{\rho}
$$

for $a \in x+\mathbb{Z}_{>0} \times \mathbb{Z}_{\geq 0}$ and $b \in x+\mathbb{Z}_{\geq 0}^{2} \times \mathbb{Z}_{>0}$. The definition above implies $I_{k e_{1}}^{x}=I_{k e_{1}}$ and $J_{l e_{2}}^{x}=J_{l e_{2}}$ for $k, l \geq 1$. The term (increment) stationary LPP is justified by the next fact. Its proof is an induction argument and can be found for example in [27, Thm. 3.1].

Lemma 3.3. Let $\left\{y_{i}\right\}$ be any finite or infinite down-right path in $x+\mathbb{Z}_{\geq 0}^{2}$. That is, $\left(y_{i+1}-y_{i}\right) \cdot e_{2} \leq 0 \leq\left(y_{i+1}-y_{i}\right) \cdot e_{1}$. Then the increments $\left\{G_{x, y_{i+1}}^{\rho}-G_{x, y_{i}}^{\rho}\right\}$ are independent. The marginal distributions of nearest-neighbor increments are $I_{a}^{x} \sim \operatorname{Exp}(1-\rho)$ and $J_{b}^{x} \sim \operatorname{Exp}(\rho)$.

Now apply Lemma 3.2 to this stationary situation. Take $z \in x+\mathbb{Z}_{\geq 0}^{2}$ and define the LPP process $G_{z, \bullet}^{(x), \rho}$ with the recipe (3.1) where the boundary weights are the ones in (3.6). By Lemma 3.3, these boundary weights have the same distribution as the original ones in (3.3). Consequently $G_{z, \bullet}^{(x), \rho}$ is another stationary LPP process. Lemma 3.2 gives the statement below which will be used extensively in our proofs.

Lemma 3.4. Let $x \leq z$ and $y \in z+\mathbb{Z}_{>0}^{2}$. Then the unique geodesics of $G_{x, y}^{\rho}$ and $G_{z, y}^{(x), \rho}$ coincide in the quadrant $z+\mathbb{Z}_{>0}^{2}$.

Since the boundary weights in (3.3) are stochastically larger than the bulk weights, the $\rho$-geodesic prefers the boundaries. The characteristic direction $\xi[\rho]=\left((1-\rho)^{2}, \rho^{2}\right)$ defined earlier in (2.2) is the unique direction in which the attraction of the $e_{1}$ - and $e_{2}$-axes balance each other out. A consequence of this is that the $\rho$-geodesic from $x$ to $x+v_{N}$ spends order $N^{2 / 3}$ steps on the boundary. Here we encounter the $2 / 3$ wandering exponent of KPZ universality. This is described in Theorems 3.5 and 4.5 below. The macroscopic picture is in Figure 3.3. This matter is discussed more thoroughly in Section 3.2 of [27]. We record the upper bound for this exit time recently derived in [14].

Theorem 3.5. [14, Theorem 2.5] There exist positive constants $r_{0}, N_{0}, C$ that depend only on $\rho$ such that for all $r>r_{0}, N \geq N_{0}$, and $\left|v-v_{N}\right|_{1} \leq N^{2 / 3}$,

$$
\mathbb{P}^{\rho}\left\{\left|\mathbf{Z}^{0 \rightarrow v}\right| \geq r N^{2 / 3}\right\} \leq e^{-C r^{3}} .
$$

In the next corollary the $\Theta\left(N^{2 / 3}\right)$ deviation is transferred from the base point 0 to the endpoint $v_{N}$. Figure 3.4 illustrates how Lemma 3.4 reduces claim (3.8) to Theorem 3.5. (Corollary 3.6 is proved using the same method as Corollary 5.10 in the arXiv version of [27].) 


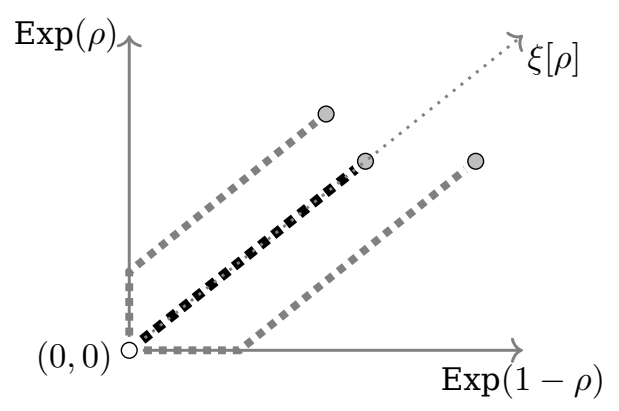

Figure 3.3: A macroscopic view of point-to-point geodesics (dotted lines) in stationary LPP from the base point at the origin $(0,0)$ to three different endpoints (gray bullets). Only the geodesic in the characteristic direction $\xi[\rho]$ spends no macroscopic time on the boundary.

$(0,0)$
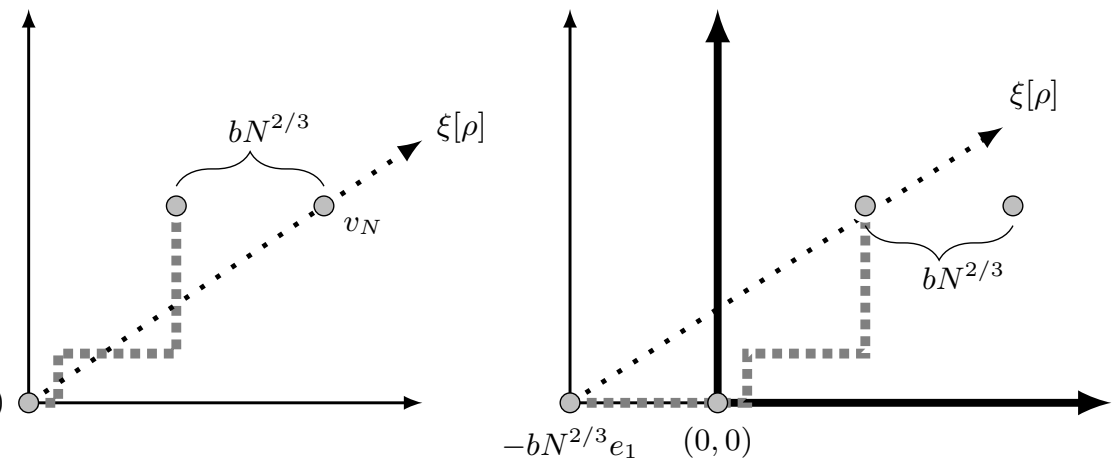

Figure 3.4: Proof of (3.8). On the left the event $\mathbf{Z}^{0 \rightarrow v_{N}-\left\lfloor b N^{2 / 3}\right\rfloor e_{1}} \geq 1$. On the right a second base point is placed at $-\left\lfloor b N^{2 / 3}\right\rfloor e_{1}$ and the increment variables on the $e_{2}$-axis based at 0 are determined by the LPP process based at $-\left\lfloor b N^{2 / 3}\right\rfloor e_{1}$. By Lemma 3.4, $\mathbf{Z}^{0 \rightarrow v_{N}-\left\lfloor b N^{2 / 3}\right\rfloor e_{1}} \geq 1$

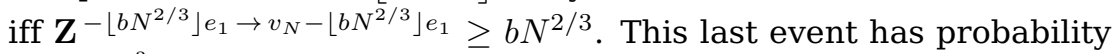
$\leq e^{-C b^{-3}}$ by Theorem 3.5.

Corollary 3.6. There exist positive constants $N_{0}, C$ that depend only on $\rho$ such that for $N \geq N_{0}$ and $b>0$,

$$
\begin{aligned}
& \mathbb{P}^{\rho}\left\{\mathbf{Z}^{0 \rightarrow v_{N}+\left\lfloor b N^{2 / 3}\right\rfloor e_{1}} \leq-1\right\} \leq e^{-C b^{3}} \quad \text { and } \\
& \mathbb{P}^{\rho}\left\{\mathbf{Z}^{0 \rightarrow v_{N}-\left\lfloor b N^{2 / 3}\right\rfloor e_{1}} \geq 1\right\} \leq e^{-C b^{3}} .
\end{aligned}
$$

\subsection{Busemann functions and semi-infinite geodesics}

The key to our results is that the directed semi-infinite geodesics can be defined through Busemann functions, which themselves are instances of stationary LPP. Thus estimates proved for stationary LPP provide information about the behavior of directed semi-infinite geodesics.

The next theorem summarizes the properties of Busemann functions needed. It is a combination of results from Section 4 of [27] and Lemma 4.1 of [28]. The dual weights introduced in part (iii) below are connected with dual geodesics which will be constructed later in Section 5. 
Theorem 3.7. Fix $\rho \in(0,1)$. Then on the probability space of the i.i.d. $\operatorname{Exp}(1)$ weights $\left\{\omega_{z}\right\}_{z \in \mathbb{Z}^{2}}$ there exists a process $\left\{B_{x, y}^{\rho}\right\}_{x, y \in \mathbb{Z}^{2}}$ with the following properties.

(i) With probability one, $\forall x, y \in \mathbb{Z}^{2}$,

$$
B_{x, y}^{\rho}=\lim _{N \rightarrow \infty}\left(G_{x, u_{N}}-G_{y, u_{N}}\right)
$$

for any sequence $u_{N}$ such that $\left|u_{N}\right| \rightarrow \infty$ and $u_{N} /\left|u_{N}\right|_{1} \rightarrow \xi[\rho] /|\xi[\rho]|_{1}$ as $N \rightarrow \infty$.

(ii) The unique $\xi[\rho]$-directed semi-infinite geodesic from $x$ is defined by $\mathbf{b}_{0}^{\rho, x}=x$ and for $k \geq 0$

$$
\mathbf{b}_{k+1}^{\rho, x}= \begin{cases}\mathbf{b}_{k}^{\rho, x}+e_{1}, & \text { if } B_{\mathbf{b}_{k}^{\rho, x}, \mathbf{b}_{k}^{\rho, x}+e_{1}}^{\rho} \leq B_{\mathbf{b}_{k}^{\rho, x}, \mathbf{b}_{k}^{\rho, x}+e_{2}}^{\rho} \\ \mathbf{b}_{k}^{\rho, x}+e_{2}, & \text { if } B_{\mathbf{b}_{k}^{\rho, x}, \mathbf{b}_{k}^{\rho, x}+e_{2}}^{\rho}<B_{\mathbf{b}_{k}^{\rho, x}, \mathbf{b}_{k}^{\rho, x}+e_{1}}^{\rho}\end{cases}
$$

(iii) Define the dual weights by

$$
\check{\omega}_{z}^{\rho}=B_{z-e_{1}, z}^{\rho} \wedge B_{z-e_{2}, z}^{\rho} \quad \text { for } z \in \mathbb{Z}^{2} .
$$

Fix a bi-infinite nearest-neighbor down-right path $\gamma=\left\{x_{i}\right\}_{i \in \mathbb{Z}}$ on $\mathbb{Z}^{2}$. This means that $x_{i+1}-x_{i} \in\left\{e_{1},-e_{2}\right\}$. Then the random variables

$$
\begin{gathered}
\left\{B_{x_{i}, x_{i+1}}^{\rho}: i \in \mathbb{Z}\right\},\left\{\omega_{y}: y \in \mathbb{Z}^{2} \text { lies strictly to the left of and below } \gamma\right\}, \\
\text { and }\left\{\breve{\omega}_{z}^{\rho}: z \in \mathbb{Z}^{2} \text { lies strictly to the right of and above } \gamma\right\}
\end{gathered}
$$

are all mutually independent with marginal distributions

$$
B_{x, x+e_{1}}^{\rho} \sim \operatorname{Exp}(1-\rho), \quad B_{x, x+e_{2}}^{\rho} \sim \operatorname{Exp}(\rho) \quad \text { and } \quad \omega_{y}, \check{\omega}_{z}^{\rho} \sim \operatorname{Exp}(1)
$$

Versions of parts (i) and (ii) above can be proved for general i.i.d. weights [17]. But nothing like part (iii) with precise distributions for Busemann functions and dual weights is known for LPP models that are not exactly solvable.

A Busemann function $B^{\rho}$ can be thought as a stationary LPP process in two ways. One with north and east boundaries, denoted by $G^{\rho, N E}$, and one with south and west boundaries, denoted by $G^{\rho}$. Here $G^{\rho}$ is as was given in (3.4), and $G^{\rho, N E}$ is defined as follows (NE stands for north and east boundaries).

Fix an origin or base point $x \in \mathbb{Z}^{2}$. Start with mutually independent bulk weights $\left\{\omega_{z}: z \in x-\mathbb{Z}_{>0}^{2}\right\}$ and boundary weights $\left\{I_{x-k e_{1}}, J_{x-l e_{2}}: k, l \in \mathbb{Z}_{\geq 0}\right\}$ with marginal distributions

$$
\omega_{z} \sim \operatorname{Exp}(1), \quad I_{x-k e_{1}} \sim \operatorname{Exp}(1-\rho), \quad \text { and } \quad J_{x-l e_{2}} \sim \operatorname{Exp}(\rho)
$$

On the boundaries define $G_{x, x}^{N E, \rho}=0, G_{x-k e_{1}, x}^{N E, \rho}=\sum_{i=0}^{k-1} I_{x-i e_{1}}$ and $G_{x+l e_{2}, x}^{N E, \rho}=\sum_{j=0}^{l-1} J_{x-j e_{2}}$ for $k, l \geq 1$. In the bulk we perform LPP that uses both the boundary and the bulk weights: for $y=x-(m, n) \in x-\mathbb{Z}_{>0}^{2}$,

$$
G_{y, x}^{N E, \rho}=\max _{1 \leq k \leq m}\left\{\left(\sum_{i=0}^{k-1} I_{x-i e_{1}}\right)+G_{y, x-k e_{1}-e_{2}}\right\} \bigvee \max _{1 \leq l \leq n}\left\{\left(\sum_{j=0}^{l-1} J_{x-j e_{2}}\right)+G_{y, x-l e_{2}-e_{1}}\right\}
$$

The LPP value $G_{a, b}$ inside the braces is the one defined by (2.1) with i.i.d. bulk weights $\omega$.

Two stationary LPP processes can be defined by taking Busemann increments as boundary weights. Fix again a base point $x \in \mathbb{Z}^{2}$. 
- Construct $G_{y, x}^{\rho, N E}$ for $y \leq x$ as in (3.11) using the NE boundary weights $I_{x-k e_{1}}=$ $B_{x-(k+1) e_{1}, x-k e_{1}}^{\rho}$ and $J_{x-l e_{2}}=B_{x-(l+1) e_{2}, x-l e_{2}}^{\rho}$ and bulk weights $\left\{\omega_{z}: z \in x-\mathbb{Z}_{>0}^{2}\right\}$.

- Construct $G_{x, y^{\prime}}^{\rho}$ for $y^{\prime} \geq x$ as in (3.4) using the SW boundary weights $I_{x+k e_{1}}=$ $B_{x+(k-1) e_{1}, x+k e_{1}}^{\rho}$ and $J_{x+l e_{2}}=B_{x+(l-1) e_{2}, x+l e_{2}}^{\rho}$ and bulk weights $\left\{\check{\omega}_{z}^{\rho}: \in x+\mathbb{Z}_{>0}^{2}\right\}$.

These two constructions satisfy the definitions of stationary LPP processes due to Theorem 3.7(iii). Their key properties relative to the Busemann function are

$$
\begin{aligned}
G_{y, x}^{\rho, N E} & =B_{y, x}^{\rho} & & \text { for all } y \leq x \\
\text { and } & G_{x, y^{\prime}}^{\rho}=B_{x, y^{\prime}}^{\rho} & & \text { for all } y^{\prime} \geq x .
\end{aligned}
$$

This is in Theorem 4.4 of [27].

As the last point, we state an independence property for a coupling of Busemann functions in two different directions. This fact was used to show the non-existence of bi-infinite geodesics [2] and local stationarity of the CGM [3]. It follows from the queuing map construction for the joint distribution (in various directions) of Busemann function from [15].

Proposition 3.8. [3, Lemma 4.5] Let $0<\eta<\lambda<1$. There exists a coupling of Busemann functions $B^{\eta}$ and $B^{\lambda}$ such that for any fixed $x \in \mathbb{Z}^{2}$ and for every $k, l \in \mathbb{Z}_{>0}$, the following sets of random variables (on the horizontal line through $x$ ) are independent:

$$
\left\{B_{x+i e_{1}, x+(i+1) e_{1}}^{\eta}\right\}_{-k \leq i \leq-1} \quad \text { and } \quad\left\{B_{x+i e_{1}, x+(i+1) e_{1}}^{\lambda}\right\}_{0 \leq i \leq l-1} .
$$

\section{Exit time estimates}

This section proves estimates on the exit time for stationary LPP processes defined in (3.4) and (3.5). These results are applied in Section 5 to prove the main theorems stated in Section 2. The first theorem is the main intermediate result towards the lower bound of Theorem 2.3. We also introduce useful lemmas that are used again later in the proof of Theorem 4.5.

Theorem 4.1. For each $0<\rho<1$ there exist finite positive constants $r_{0}(\rho), C(\rho)$ and $N_{0}(\rho)$ such that for all $N \geq N_{0}(\rho)$ and $r_{0} \leq r \leq\left[(1-\rho)^{2} \wedge \rho^{2}\right] N^{1 / 3}$,

$$
\mathbb{P}^{\rho}\left\{\forall z \text { outside } \llbracket 0, v_{N} \rrbracket \text { we have }\left|\mathbf{Z}^{0 \rightarrow z}\right| \geq r N^{2 / 3}\right\} \geq e^{-C r^{3}} .
$$

To prove this bound we tilt the probability measure to make the event likely and pay for this with a moment bound on the Radon-Nikodym derivative. This argument was introduced in [6] in the context of ASEP, and adapted to a lower bound proof of the longitudinal fluctuation exponent in the stationary LPP in Section 5.5 of the lectures [27]. The key idea is a perturbation of the parameter $\rho$ of the stationary LPP process to $\rho \pm r N^{-1 / 3}$. This allows us to control the exit point on the scale $N^{2 / 3}$. The general idea of utilizing perturbations of order $N^{-1 / 3}$ goes back to the seminal paper [9].

Lemma 4.2 below is an auxiliary estimate for the proof of Theorem 4.1. It utilizes a perturbed parameter $\lambda=\rho+r N^{-1 / 3}$, assumed to satisfy

$$
\rho<\lambda \leq c(\rho)<1
$$

for some constant $c(\rho)<1$, as $r$ and $N$ vary. Lemma 4.2 shows that, for small enough $a>0$ and large enough $b, r>0$, the $\lambda$-geodesic to a target point $w_{N}$ slightly perturbed from $v_{N}$ exits the $e_{1}$-axis through the interval $\llbracket a r N^{2 / 3} e_{1}, b r N^{2 / 3} e_{1} \rrbracket$ with high probability. This is illustrated on the right of Figure 4.1. The constants $1-\rho$ and $2 / \rho^{2}$ in Lemma 4.2 come from the following observation (left diagram of Figure 4.1$)$. Start two rays at $(0,0)$ 

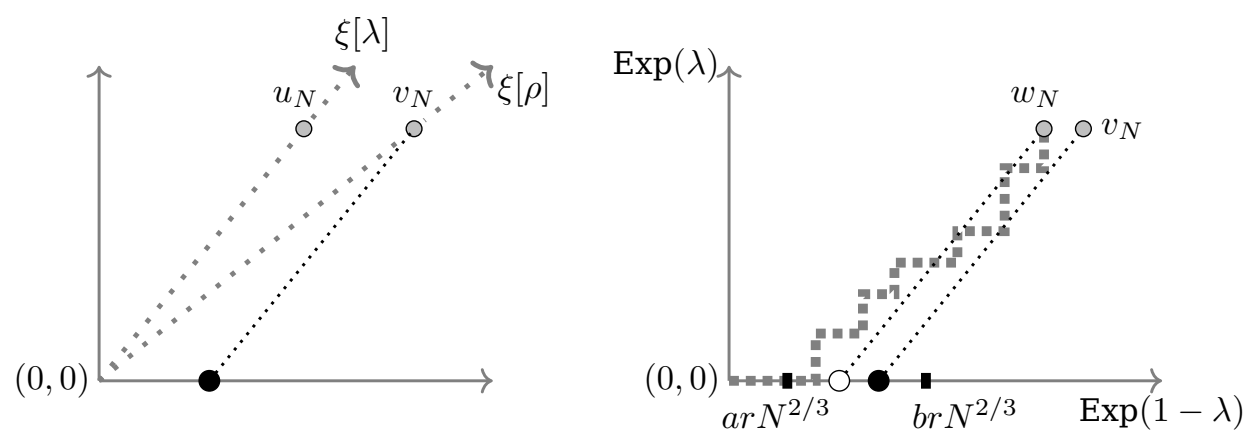

Figure 4.1: Left: Illustration of estimate (4.2). Right: Illustration of Lemma 4.2. The dotted lines have characteristic slope $\xi[\lambda]$. Consequently, with high probability, the geodesic from 0 to $w_{N}$ exits through the interval $\llbracket a r N^{2 / 3} e_{1}, b r N^{2 / 3} e_{1} \rrbracket$.

in the directions $\xi[\rho]$ and $\xi[\lambda]$ and let $u_{N}$ be the lattice point closest to the $\xi[\lambda]$-directed ray such that $u_{N} \cdot e_{2}=v_{N} \cdot e_{2}$. Then

$$
(1-\rho) r N^{2 / 3} \leq v_{N} \cdot e_{1}-u_{N} \cdot e_{1} \leq \frac{2}{\rho^{2}} r N^{2 / 3}
$$

Lemma 4.2. Let $\lambda=\rho+r N^{-1 / 3}$ and $w_{N}=v_{N}-\left\lfloor\frac{1}{10}(1-\rho) r N^{2 / 3}\right\rfloor e_{1}$. There exist positive constants $C, N_{0}$ that depend only on $\rho$ such that, for any $r>0$ and $N \geq N_{0}$ such that (4.1) holds, we have

$$
\mathbb{P}^{\lambda}\left(\frac{1}{10}(1-\rho) r N^{2 / 3} \leq \mathbf{Z}^{0 \rightarrow w_{N}} \leq 10 \frac{2}{\rho^{2}} r N^{2 / 3}\right) \geq 1-e^{-C r^{3}}
$$

Before the proof of Lemma 4.2, we separate an observation about geodesics in the next lemma, illustrated by the left diagram of Figure 4.2. It comes from the idea of Lemma 3.2 of constructing nested LPP processes with boundary weights defined by increments of an outer LPP process. (Lemma 4.3 is proved as Lemma A.3 in the appendix of [27].)

Lemma 4.3. Fix two base points $(0,0)$ and $(m,-n)$ with $m, n>0$. From these base

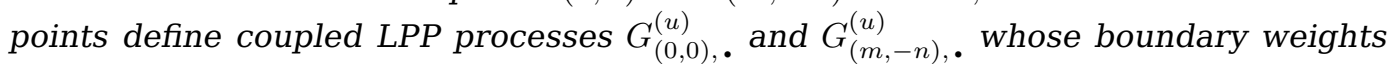
come from the increments of an LPP process $G_{u, .}$ whose base point $u$ satisfies $u \leq(0,0)$ and $u \leq(m,-n)$. Then for $z \in\left((0,0)+\mathbb{Z}_{>0}^{2}\right) \cap\left((m,-n)+\mathbb{Z}_{>0}^{2}\right), \mathbf{Z}^{0 \rightarrow z} \leq m$ if and only if $\mathbf{Z}^{(m,-n)} \rightarrow z<-n$.

Proof of Lemma 4.2. Let $a=\frac{1}{10}(1-\rho), b=10 \frac{2}{\rho^{2}}$.

It suffices to show that if $r>0$ and $N \geq N_{0}$ are such that (4.1) holds, then

$$
\begin{aligned}
& \mathbb{P}^{\lambda}\left(\mathbf{Z}^{0 \rightarrow w_{N}}<a r N^{2 / 3}\right) \leq e^{-C r^{3}} \\
& \mathbb{P}^{\lambda}\left(\mathbf{Z}^{0 \rightarrow w_{N}}>b r N^{2 / 3}\right) \leq e^{-C r^{3}} .
\end{aligned}
$$

By (4.2) the distance between the origin and the black dot on the $x$-axis on the right of Figure 4.1 is bounded above by $\frac{2}{\rho^{2}} r N^{2 / 3}=\frac{1}{10} b r N^{2 / 3}$. So the distance between the black dot and $b r N^{2 / 3} e_{1}$ is at least $b r N^{2 / 3}-\frac{1}{10} b r N^{2 / 3}=\frac{9}{10} b r N^{2 / 3}$. Apply Lemma 3.4 to switch from the geodesic based at the origin to one based at the black dot, and apply 

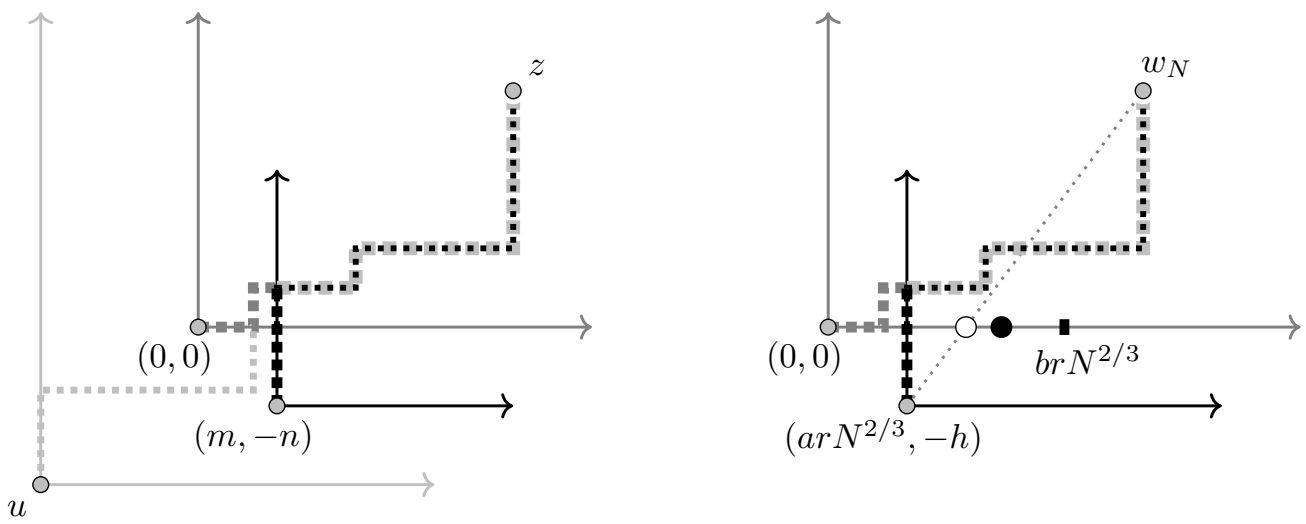

Figure 4.2: Left: An illustration of Lemma 4.3. As shown in the picture $\mathbf{Z}^{(0,0) \rightarrow z} \leq m$ if and only if $\mathbf{Z}^{(m,-n) \rightarrow z}<-n$. Right: Applying Lemma 4.3 in the proof of Lemma 4.2 to assert that $\mathbb{P}^{\lambda}\left(\mathbf{Z}^{0 \rightarrow w_{N}} \leq\left\lfloor\right.\right.$ ar $\left.\left.N^{2 / 3}\right\rfloor\right)=$ $\mathbb{P}^{\lambda}\left(\mathbf{Z}^{\left(\left\lfloor a r N^{2 / 3}\right\rfloor,-h\right) \rightarrow w_{N}}<-h\right)$.

Theorem 3.5 to the LPP process $G_{\text {blackdot, }}^{(0),}$ :

$$
\begin{gathered}
\mathbb{P}^{\lambda}\left(\mathbf{Z}^{0 \rightarrow w_{N}}>b r N^{2 / 3}\right) \leq \mathbb{P}^{\lambda}\left(\mathbf{Z}^{0 \rightarrow v_{N}}>b r N^{2 / 3}\right) \\
\leq \mathbb{P}^{\lambda}\left(\mathbf{Z}^{\text {black dot } \rightarrow v_{N}} \geq \frac{9}{10} b r N^{2 / 3}\right) \leq e^{-C r^{3}}
\end{gathered}
$$

To prove (4.3) choose $h$ so that $\left(\left\lfloor a r N^{2 / 3}\right\rfloor,-h\right)$ is the closest integer point to the $\left(-\xi[\lambda]\right.$ )-directed ray starting at $w_{N}$ (see Figure 4.2 ). Lemma 4.3 gives

$$
\mathbb{P}^{\lambda}\left(\mathbf{Z}^{0 \rightarrow w_{N}} \leq\left\lfloor a r N^{2 / 3}\right\rfloor\right)=\mathbb{P}^{\lambda}\left(\mathbf{Z}^{\left(\left\lfloor a r N^{2 / 3}\right\rfloor,-h\right) \rightarrow w_{N}}<-h\right) .
$$

Theorem 3.5 states that it is unlikely for the $\lambda$-geodesic from $\left(\left\lfloor a r N^{2 / 3}\right\rfloor,-h\right)$ to $w_{N}$ to exit late in the scale $N^{2 / 3}$ from the $y$-axis, because the direction is the characteristic one $\xi[\lambda]$. Thus it suffices to show $h$ is bounded below by some $k(\rho) r N^{2 / 3}$.

Using the lower bound from (4.2), the distance between the black dot and $(0,0)$ is bounded below by $(1-\rho) r N^{2 / 3}=10 a r N^{2 / 3}$. The distance between the black dot and $\left\lfloor a r N^{2 / 3}\right\rfloor e_{1}$ is bounded below by $9 a r N^{2 / 3}$, and the distance between the white dot and $\left\lfloor a r N^{2 / 3}\right\rfloor e_{1}$ is bounded below by $8 a r N^{2 / 3}$. The slope of the line going through $w_{N}$ and white dot is $\frac{\lambda^{2}}{(1-\lambda)^{2}}$. Thus, we have

$$
h \geq \frac{\lambda^{2}}{(1-\lambda)^{2}} \operatorname{sarN}^{2 / 3} .
$$

Since $\lambda$ is bounded above and below by constants that depend on $\rho$, we get

$$
h \geq k(\rho) r N^{2 / 3}
$$

which finishes the proof.

Proof of Theorem 4.1. For two fixed constants $0<a<b$, we increase the weights on the intervals $\llbracket\left\lfloor a r N^{2 / 3}\right\rfloor e_{1},\left\lfloor b r N^{2 / 3}\right\rfloor e_{1} \rrbracket$ and $\llbracket\left\lfloor a r N^{2 / 3}\right\rfloor e_{2},\left\lfloor b r N^{2 / 3}\right\rfloor e_{2} \rrbracket$. The new weights are chosen so that their characteristic directions obey the left diagram of Figure 4.3 for large $N \geq N_{0}(\rho)$. 


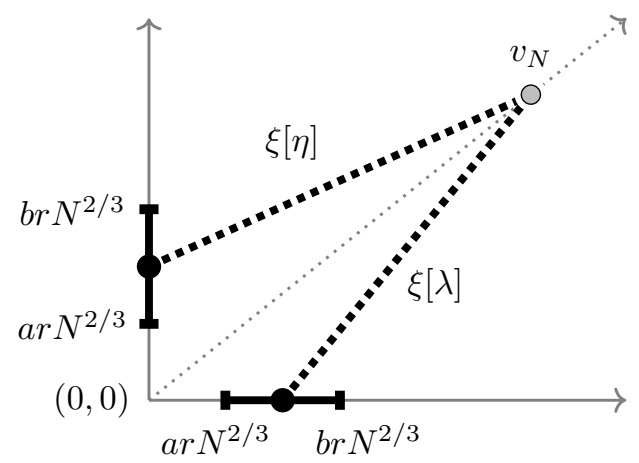

$(0,0)$

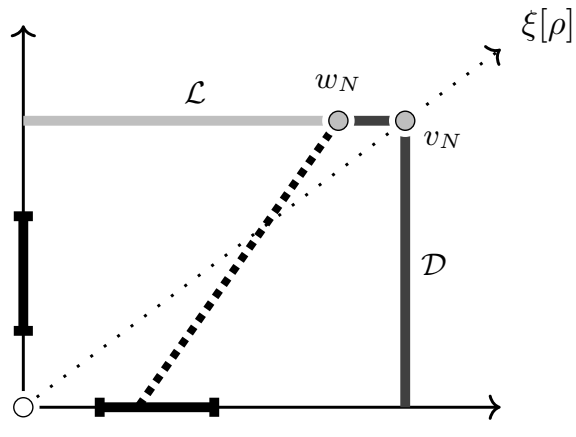

Figure 4.3: Left: Two dotted lines have slopes $\xi[\lambda]$ and $\xi[\eta]$. right: Decomposition of the north and east boundaries of $\llbracket 0, v_{N} \rrbracket$ into regions $\mathcal{L}$ (light gray) and $\mathcal{D}$ (dark gray). A small perturbation of $v_{N}$ to $w_{N}$ keeps the endpoint of the $-\xi[\lambda]$ ray from $w_{N}$ in the interval $\left[a r N^{2 / 3}, b r N^{2 / 3}\right]$.

On the $e_{1}$-axis, define

$$
\lambda=\rho+\frac{r}{N^{1 / 3}} .
$$

The assumption $0<r \leq\left[(1-\rho)^{2} \wedge \rho^{2}\right] N^{1 / 3}$ guarantees that $0<\lambda \leq \rho+(1-\rho)^{2}<1$. Use $\operatorname{Exp}(1-\lambda)$ as the heavier weights and pick

$$
a=\frac{1}{10}(1-\rho), \quad b=10 \frac{2}{\rho^{2}}
$$

as in Lemma 4.2.

On the $e_{2}$-axis, we define

$$
\eta=\rho-\frac{r}{N^{1 / 3}},
$$

and the heavier weights are $\operatorname{Exp}(\eta)$. The condition $0<r \leq\left[(1-\rho)^{2} \wedge \rho^{2}\right] N^{1 / 3}$ guarantees that $0<\rho-(1-\rho)^{2} \wedge \rho^{2} \leq \eta<\rho$. Note that Lemma 4.2 continues to hold if $a$ is decreased and $b$ is increased. The constants $a, b, N_{0}$ can always be adjusted so that the situation in the left diagram of Figure 4.3 appears.

Recall the old environment of the stationary $\rho$-LPP process:

$$
\begin{aligned}
\omega_{z} & \sim \operatorname{Exp}(1) & & \text { for } z \in \mathbb{Z}_{>0}^{2} \\
\omega_{k e_{1}} & \sim \operatorname{Exp}(1-\rho) & & \text { for } k \geq 1 \\
\omega_{l e_{2}} & \sim \operatorname{Exp}(\rho) & & \text { for } l \geq 1 .
\end{aligned}
$$

The new environment $\widetilde{\omega}$ increases the weights in the two intervals on the axes:

$$
\begin{aligned}
\widetilde{\omega}_{z} & =\omega_{z} & & \text { for } z \quad \notin \llbracket\left\lfloor a r N^{2 / 3}\right\rfloor e_{1},\left\lfloor b r N^{2 / 3}\right\rfloor e_{1} \rrbracket \cup \llbracket\left\lfloor a r N^{2 / 3}\right\rfloor e_{2},\left\lfloor b r N^{2 / 3}\right\rfloor e_{2} \rrbracket \\
\widetilde{\omega}_{k e_{1}} & =\frac{1-\rho}{1-\lambda} \omega_{k e_{1}} & & \text { for } k e_{1} \in \llbracket\left\lfloor a r N^{2 / 3}\right\rfloor e_{1},\left\lfloor b r N^{2 / 3}\right\rfloor e_{1} \rrbracket \\
\widetilde{\omega}_{l e_{2}} & =\frac{\rho}{\eta} \omega_{l e_{2}} & & \text { for } l e_{2} \in \llbracket\left\lfloor a r N^{2 / 3}\right\rfloor e_{2},\left\lfloor b r N^{2 / 3}\right\rfloor e_{2} \rrbracket .
\end{aligned}
$$

Denote the probability measure for the environment $\widetilde{\omega}$ by $\widetilde{\mathbb{P}}$. The goal is the estimate

$$
\widetilde{\mathbb{P}}(A) \equiv \widetilde{\mathbb{P}}\left\{\forall z \text { outside } \llbracket 0, v_{N} \rrbracket \text { we have }\left|\mathbf{Z}^{0 \rightarrow z}\right| \geq \operatorname{ar} N^{2 / 3}\right\} \geq 1 / 2
$$


where $A$ denotes the event in braces. We check that this implies Theorem 4.1. The Cauchy-Schwartz inequality gives

$$
1 / 2 \leq \widetilde{\mathbb{P}}(A)=\mathbb{E}^{\rho}\left[\mathbf{1}_{A} f_{N}\right] \leq\left(\mathbb{P}^{\rho}(A)\right)^{1 / 2}\left(\mathbb{E}^{\rho}\left[f^{2}\right]\right)^{1 / 2}
$$

where $f$ is the Radon-Nikodym derivative. Lemma A.2 gives the bound

$$
\mathbb{E}^{\rho}\left[f^{2}\right] \leq e^{C r^{3}}
$$

and then (4.6) and (4.7) imply the lower bound

$$
\mathbb{P}^{\rho}(A) \geq \frac{1}{2} e^{-C r^{3}} .
$$

Note that the event $A$ in (4.5) has the lower bound $\geq a r N^{2 / 3}$. To replace this with $\geq r N^{2 / 3}$, as required for Theorem 4.1, modify the constant $C$.

To show (4.5) we bound its complement:

$$
\widetilde{\mathbb{P}}\left\{\exists z \text { outside } \llbracket 0, v_{N} \rrbracket \text { such that }\left|\mathbf{Z}^{0 \rightarrow z}\right| \leq \operatorname{ar} N^{2 / 3}\right\} \leq e^{-C r^{3}} .
$$

We treat the case $1 \leq \mathbf{Z}^{0 \rightarrow z} \leq a r N^{2 / 3}$ of (4.8). The same arguments give the analogous bound for the case $-a r N^{2 / 3} \leq \mathbf{Z} \leq-1$. Define $w_{N}=v_{N}-\left\lfloor\frac{1}{10}(1-\rho) r N^{2 / 3}\right\rfloor e_{1}$, and break up the northeast boundary of $\llbracket 0, v_{N} \rrbracket$ into two regions $\mathcal{L}$ and $\mathcal{D}$ as in the diagram on the right of Figure 4.3.

First consider geodesics that hit $\mathcal{D}$. Let $\sigma_{1}^{0 \rightarrow x}$ denote the exit time of the optimal $0 \rightarrow x$ path among those paths whose first step is $e_{1}$.

$$
\begin{aligned}
\widetilde{\mathbb{P}}\{\exists & \left.\in \mathcal{D}: 1 \leq \mathbf{Z}^{0 \rightarrow z}<\operatorname{ar} N^{2 / 3}\right\} \leq \widetilde{\mathbb{P}}\left\{\exists z \in \mathcal{D}: \sigma_{1}^{0 \rightarrow z}<\operatorname{ar} N^{2 / 3}\right\} \\
& \leq \widetilde{\mathbb{P}}\left\{\sigma_{1}^{0 \rightarrow w_{N}}<a r N^{2 / 3}\right\} \leq \widetilde{\mathbb{P}}\left\{\sigma_{1}^{0 \rightarrow w_{N}} \notin \llbracket\left\lfloor a r N^{2 / 3}\right\rfloor e_{1},\left\lfloor b r N^{2 / 3}\right\rfloor e_{1} \rrbracket\right\} \\
& \leq \mathbb{P}^{\lambda}\left\{\sigma_{1}^{0 \rightarrow w_{N}} \notin \llbracket\left\lfloor a r N^{2 / 3}\right\rfloor e_{1},\left\lfloor b r N^{2 / 3}\right\rfloor e_{1} \rrbracket\right\} \\
& \leq \mathbb{P}^{\lambda}\left\{\mathbf{Z}^{0 \rightarrow w_{N}} \notin \llbracket\left\lfloor a r N^{2 / 3}\right\rfloor e_{1},\left\lfloor b r N^{2 / 3}\right\rfloor e_{1} \rrbracket\right\} \leq e^{-C r^{3}} .
\end{aligned}
$$

The second inequality comes from the uniqueness of maximizing paths: the maximizing path to $w_{N}$ cannot go to the right of a maximizing path to $\mathcal{D}$. The switch from $\widetilde{\mathbb{P}}$ to $\mathbb{P}^{\lambda}$ increases the boundary weights on the $e_{1}$ axis outside the interval $\llbracket\left\lfloor a r N^{2 / 3}\right\rfloor e_{1},\left\lfloor b r N^{2 / 3}\right\rfloor e_{1} \rrbracket$, hence the fourth inequality. The last inequality is from Lemma 4.2.

Consider the light gray region $\mathcal{L}$. The switch from $\widetilde{\mathbb{P}}$ to $\mathbb{P}^{\rho}$ decreases certain boundary weights outside the range $\llbracket e_{1},\left\lceil a r N^{2 / 3}-1\right\rceil e_{1} \rrbracket$ and gives the first inequality below.

$$
\begin{gathered}
\widetilde{\mathbb{P}}\left\{\exists z \in \mathcal{L}: 1 \leq \mathbf{Z}^{0 \rightarrow z}<\operatorname{ar} N^{2 / 3}\right\} \leq \mathbb{P}^{\rho}\left\{\exists z \in \mathcal{L}: 1 \leq \mathbf{Z}^{0 \rightarrow z}<\operatorname{arN} N^{2 / 3}\right\} \\
\leq \mathbb{P}^{\rho}\left\{\exists z \in \mathcal{L}: \mathbf{Z}^{0 \rightarrow z} \geq 1\right\} \leq \mathbb{P}^{\rho}\left\{\mathbf{Z}^{0 \rightarrow w_{N}} \geq 1\right\} \leq e^{-C r^{3}} .
\end{gathered}
$$

The last inequality follows from bound (3.8) in Corollary 3.6.

Combining (4.9) and (4.10) gives

$$
\widetilde{\mathbb{P}}\left\{\exists z \text { outside } \llbracket 0, v_{N} \rrbracket \text { such that } 1 \leq \mathbf{Z}^{0 \rightarrow z} \leq a r N^{2 / 3}\right\} \leq e^{-C r^{3}} .
$$

The proof is complete.

The next theorem is the main intermediate result towards the lower bound of Theorem 2.2.

Theorem 4.4. For each $0<\rho<1$ there exist finite positive constants $\delta_{0}(\rho), C(\rho)$ and $N_{0}(\rho)$ such that for all $N \geq N_{0}(\rho)$ and $N^{-2 / 3} \leq \delta \leq \delta_{0}(\rho)$,

$$
\mathbb{P}^{\rho}\left\{\exists z \text { outside } \llbracket 0, v_{N} \rrbracket \text { such that }\left|\mathbf{Z}^{0 \rightarrow z}\right| \leq \delta N^{2 / 3}\right\} \geq C(\rho) \delta .
$$




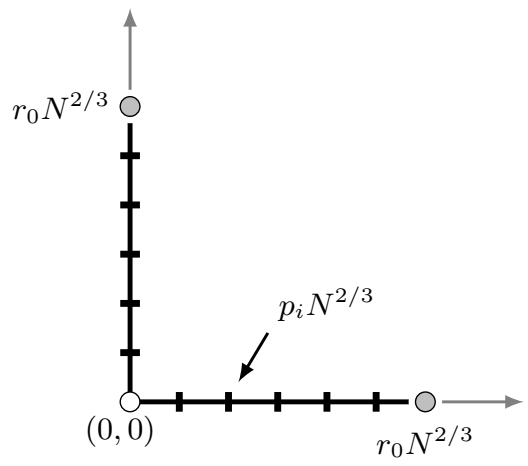

Figure 4.4: Partition of the range of $\mathbf{Z}^{0 \rightarrow v_{N}}$ in the event in (4.11). The origin is not necessarily a partition point.

Proof. Utilizing Theorem 3.5, fix constants $r_{0}, C_{0}$ and $N_{0}$ (depending on $\rho$ ) such that, for $N \geq N_{0}$,

$$
\mathbb{P}^{\rho}\left\{\left|\mathbf{Z}^{0 \rightarrow v_{N}+e_{1}+e_{2}}\right| \leq r_{0} N^{2 / 3}\right\} \geq 1 / 2 .
$$

Set $v_{N}^{\prime}=v_{N}+e_{1}+e_{2}$. Given small $\delta>N^{-2 / 3}$, partition $\left[-r_{0}, r_{0}\right]$ as

$$
-r_{0}=p_{0}<p_{1}<\cdots<p_{\left\lfloor\frac{2 r_{0}}{\delta}\right\rfloor}<p_{\left\lfloor\frac{2 r_{0}}{\delta}\right\rfloor+1}=r_{0}
$$

with mesh $p_{i+1}-p_{i} \leq \delta$. See Figure 4.4. By (4.11) there exists an integer $i^{\star} \in\left[0,\left\lfloor\frac{2 r_{0}}{\delta}\right\rfloor\right]$ such that

$$
\mathbb{P}^{\rho}\left\{p_{i^{\star}} N^{2 / 3} \leq \mathbf{Z}^{0 \rightarrow v_{N}^{\prime}} \leq p_{i^{\star}+1} N^{2 / 3}\right\} \geq \frac{\frac{1}{2} \delta}{2 r_{0}}=C(\rho) \delta .
$$

We cannot control the exact location of $i^{\star}$. We compensate by varying the endpoint around $v_{N}^{\prime}$. Let

$$
A_{N}=\llbracket v_{N}^{\prime}-r_{0} N^{2 / 3} e_{1}, v_{N}^{\prime} \rrbracket \cup \llbracket v_{N}^{\prime}-r_{0} N^{2 / 3} e_{2}, v_{N}^{\prime} \rrbracket
$$

denote the set of lattice points on the boundary of the rectangle $\llbracket 0, v_{N}^{\prime} \rrbracket$ within distance $r_{0} N^{2 / 3}$ of the upper right corner $v_{N}^{\prime}$. We claim that for any integer $i \in\left[0,\left\lfloor\frac{2 r_{0}}{\delta}\right\rfloor\right]$,

$$
\mathbb{P}^{\rho}\left\{\exists z \in A_{N}:\left|\mathbf{Z}^{0 \rightarrow z}\right| \leq \delta N^{2 / 3}\right\} \geq \mathbb{P}^{\rho}\left\{p_{i} N^{2 / 3} \leq \mathbf{Z}^{0 \rightarrow v_{N}^{\prime}} \leq p_{i+1} N^{2 / 3}\right\} .
$$

Then bounds (4.12) and (4.13) imply

$$
\mathbb{P}^{\rho}\left\{\exists z \in A_{N}:\left|\mathbf{Z}^{0 \rightarrow z}\right| \leq \delta N^{2 / 3}\right\} \geq C(\rho) \delta,
$$

and Theorem 4.4 directly follows from (4.14).

We prove claim (4.13). If $p_{i} \leq 0 \leq p_{i+1}$, (4.13) is immediate. We argue the case $p_{i+1}>p_{i}>0$, the other one being analogous. Set $z=\left(\left\lfloor p_{i} N^{2 / 3}\right\rfloor-1\right) e_{1}$ and apply Lemma 3.4 to the LPP process $G_{z, \bullet}^{(0), \rho}$. Then

$$
\begin{aligned}
\mathbb{P}^{\rho}\left\{p_{i} N^{2 / 3} \leq \mathbf{Z}^{0 \rightarrow v_{N}^{\prime}} \leq p_{i+1} N^{2 / 3}\right\} & \leq \mathbb{P}^{\rho}\left\{1 \leq \mathbf{Z}^{0 \rightarrow v_{N}^{\prime}-\left(\left\lfloor p_{i} N^{2 / 3}\right\rfloor-1\right) e_{1}} \leq \delta N^{2 / 3}\right\} \\
& \leq \mathbb{P}^{\rho}\left\{\exists z \in A_{N}:\left|\mathbf{Z}^{0 \rightarrow z}\right| \leq \delta N^{2 / 3}\right\} .
\end{aligned}
$$

The remainder of this section proves the main intermediate result towards the upper bound of Theorem 2.2. It quantifies the lower bound on the exit point on the scale $N^{2 / 3}$. This strengthens the estimates accessible without integrable probability, for previously no quantification was attained (Theorem 2.2(b) in [4]). The proof is based on the ideas from the recent work of $[2,3]$. 


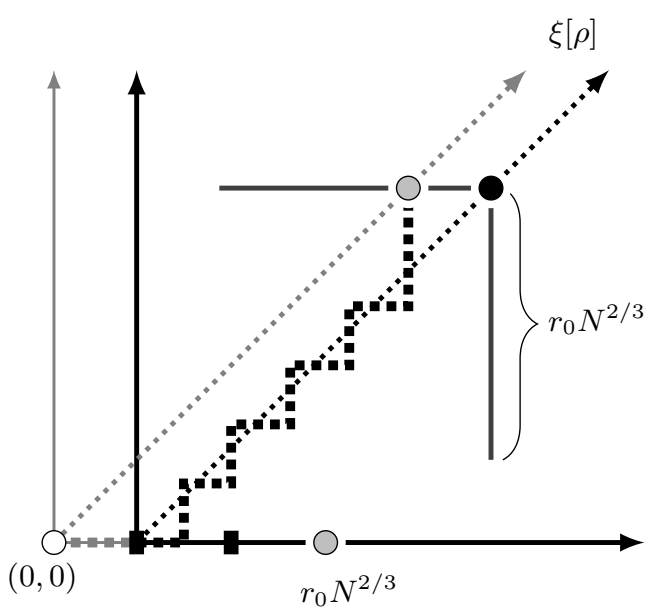

Figure 4.5: The setup for proving (4.13).

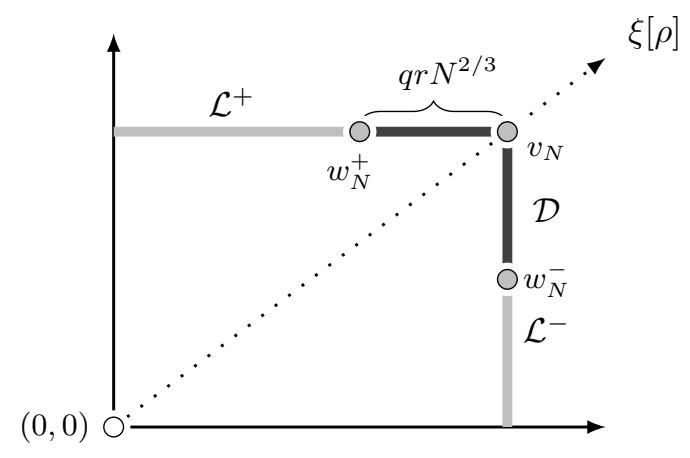

Figure 4.6: The north and east boundaries of $\llbracket 0, v_{N} \rrbracket$ are decomposed into $\mathcal{L}^{ \pm}$(light gray) and $\mathcal{D}$ (dark gray). The parameter $q$ is less than some small constant that depends only on $\rho$.

Theorem 4.5. For each $0<\rho<1$ there exist finite positive constants $\delta_{0}(\rho), C(\rho)$ and $N_{0}(\rho)$ such that for all $0<\delta \leq \delta_{0}(\rho)$ and $N \geq N_{0}(\rho)$,

$$
\mathbb{P}^{\rho}\left\{\exists z \text { outside } \llbracket 0, v_{N} \rrbracket \text { such that }\left|\mathbf{Z}^{0 \rightarrow z}\right| \leq \delta N^{2 / 3}\right\} \leq C|\log \delta|^{2 / 3} \delta \text {. }
$$

Proof. We prove the case $1 \leq \mathbf{Z} \leq \delta N^{2 / 3}$. The proof for $-\delta N^{2 / 3} \leq \mathbf{Z} \leq-1$ is similar. It suffices to look at the north and east boundaries of $\llbracket 0, v_{N} \rrbracket$ since any geodesic from 0 to outside of $\llbracket 0, v_{N} \rrbracket$ crosses the boundary. Decompose these boundaries into three parts $\mathcal{D}$ and $\mathcal{L}^{ \pm}$as in Figure 4.6, with

$$
w_{N}^{+}=v_{N}-\left\lfloor q r N^{2 / 3}\right\rfloor e_{1} \quad \text { and } \quad w_{N}^{-}=v_{N}-\left\lfloor q r N^{2 / 3}\right\rfloor e_{2}
$$

where $q$ is a small positive constant chosen later, and $r=(|\log \delta| / C)^{1 / 3}$ where $C$ is the constant in the right-hand side of the estimate in Theorem 3.5. The dark gray set $\mathcal{D}$ comprises the vertices between $w_{N}^{+}$and $w_{N}^{-}$in the north-east corner of the boundary of the rectangle $\llbracket 0, v_{N} \rrbracket$. 


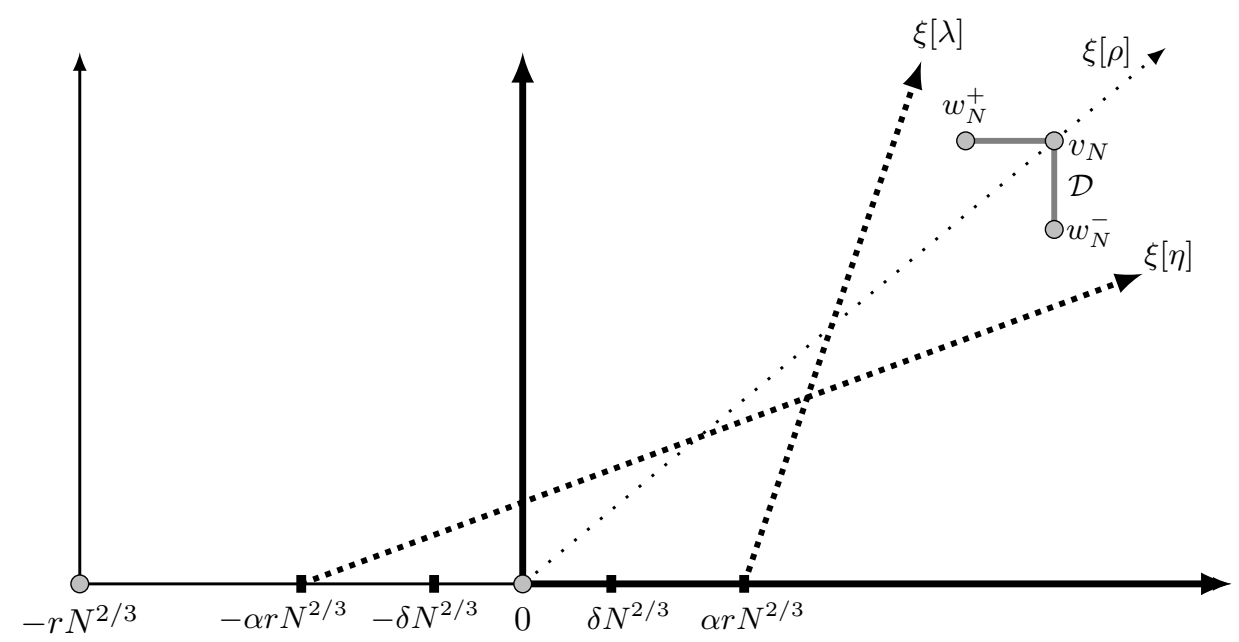

Figure 4.7: Illustration of the set $\mathcal{D}$, the nested LPP processes, and three characteristic directions. The parameters $q=\alpha$ are less than some small constant that depends only on $\rho, \delta$ is a small positive constant in $\left(0, \delta_{0}\right)$, and $r$ is a large constant with $r=(|\log \delta| / C)^{1 / 3}$.

Consider first, the dark gray portion $\mathcal{D}$. Take $0<\delta \leq \delta_{0}=\frac{9}{10}$, where the bound $\frac{9}{10}$ may be decreased later in the proof. Our goal is to estimate

$$
\mathbb{P}^{\rho}\left\{\exists z \in \mathcal{D} \text { such that } 1 \leq \mathbf{Z}^{0 \rightarrow z} \leq \delta N^{2 / 3}\right\} .
$$

To do this, we place the stationary LPP process on $0+\mathbb{Z}_{>0}^{2}$ as a nested LPP process inside a larger stationary LPP process on the quadrant $-\left\lfloor r N^{2 / 3}\right\rfloor e_{1}+\mathbb{Z}_{\geq 0}^{2}$, as shown in Figure 4.7. From the relation between geodesics of two nested LPP processes given in Lemma 3.4,

$$
\begin{aligned}
& \mathbb{P}^{\rho}\left\{\exists z \in \mathcal{D}: 1 \leq \mathbf{Z}^{0 \rightarrow z} \leq \delta N^{2 / 3}\right\} \\
& \quad \leq \mathbb{P}^{\rho}\left\{\exists z \in \mathcal{D}:\left\lfloor r N^{2 / 3}\right\rfloor-\delta N^{2 / 3} \leq \mathbf{Z}^{-\left\lfloor r N^{2 / 3}\right\rfloor e_{1} \rightarrow z} \leq\left\lfloor r N^{2 / 3}\right\rfloor+\delta N^{2 / 3}\right\}
\end{aligned}
$$

Thus, it suffices to obtain an upper bound for the second line above. To continue, we describe the rest of the setup shown in Figure 4.7.

The probability in (4.15) vanishes if $\delta N^{2 / 3}<1$ and hence we can always assume

$$
N \geq \delta^{-3 / 2}
$$

Introduce the perturbed parameters

$$
\lambda=\rho+\frac{r}{N^{1 / 3}} \quad \text { and } \quad \eta=\rho-\frac{r}{N^{1 / 3}} .
$$

We require the following bounds to hold for these two parameters

$$
\rho<\lambda \leq \rho+\frac{\rho \wedge(1-\rho)}{2}<1 \quad \text { and } \quad 0<\rho-\frac{\rho \wedge(1-\rho)}{2} \leq \eta<\rho .
$$

The point of the choice $\rho \pm \frac{\rho \wedge(1-\rho)}{2}$ is only to bound $\lambda$ and $\eta$ from above and below by two constants strictly inside $(0,1)$ and that depend only on $\rho$. These two requirements can be rewritten as

$$
N \geq\left(\frac{2 r}{\rho \wedge(1-\rho)}\right)^{3}
$$


With (4.16), this bound on $N$ is automatically satisfied as long as $\delta^{-3 / 2} \geq\left(\frac{2 r}{\rho \wedge(1-\rho)}\right)^{3}$. With $r=\left(\frac{|\log \delta|}{C}\right)^{1 / 3}$, we can ensure this by considering $\delta>0$ subject to

$$
\delta \leq \delta_{0}(\rho)=\left(\frac{1}{2} C(\rho \wedge(1-\rho))\right)^{3} \wedge \frac{9}{10}
$$

Our next step is to fix $q$ and $\alpha$ small enough so that the $\xi[\eta]$ - and $\xi[\lambda]$-directed rays started at the points $\pm\left\lfloor\alpha r N^{2 / 3}\right\rfloor e_{1}$ avoid $\mathcal{D}$ as shown in Figure 4.7. As in Figure 4.1, let $u_{N}$ be the lattice point closest to where the $\xi[\lambda]$-ray from the origin crosses the north boundary of $\llbracket 0, v_{N} \rrbracket$. Then from (4.2) we have

$$
v_{N} \cdot e_{1}-u_{N} \cdot e_{1} \geq(1-\rho) r N^{2 / 3} .
$$

Shift the starting point of the $\xi[\lambda]$-ray from the origin to $\left\lfloor\alpha r N^{2 / 3}\right\rfloor e_{1}$, and let $u_{N}^{\prime}$ be the new crossing point on the north boundary of $\llbracket 0, v_{N} \rrbracket$. By picking $q=\alpha=\frac{1-\rho}{10}$, the following lower bound holds:

$$
w_{N}^{+} \cdot e_{1}-u_{N}^{\prime} \cdot e_{1} \geq \frac{1-\rho}{2} r N^{2 / 3}
$$

This gives us the desired picture for $\xi[\lambda]$ shown in Figure 4.7. The argument for the $\xi[\eta]$-directed ray is similar. We may need to decrease $\alpha$ and $q$ further to achieve this but their values depend only on $\rho$. At last, once $\alpha$ is fixed, $r=\left(\frac{|\log \delta|}{C}\right)^{1 / 3}$ allows us to decrease $\delta_{0}$ further so that $\delta<\frac{1}{3} \alpha r$ for each $0<\delta \leq \delta_{0}$. This completes the description of the setup in Figure 4.7.

Now, to bound

$$
\mathbb{P}^{\rho}\left\{\exists z \in \mathcal{D}:\left\lfloor r N^{2 / 3}\right\rfloor-\delta N^{2 / 3} \leq \mathbf{Z}^{-\left\lfloor r N^{2 / 3}\right\rfloor e_{1} \rightarrow z} \leq\left\lfloor r N^{2 / 3}\right\rfloor+\delta N^{2 / 3}\right\},
$$

we first bound the probability

$$
\mathbb{P}^{\rho}\left\{\exists z \in \mathcal{D}: \mathbf{Z}^{-\left\lfloor r N^{2 / 3}\right\rfloor e_{1} \rightarrow z}=\left\lfloor r N^{2 / 3}\right\rfloor+t_{0}\right\}
$$

where $t_{0}$ is a fixed integer in $\llbracket-\left\lfloor\delta N^{2 / 3}\right\rfloor,\left\lfloor\delta N^{2 / 3}\right\rfloor \rrbracket$.

For $z \in \mathcal{D}$ and $i \in \llbracket-\left\lfloor\alpha r N^{2 / 3}\right\rfloor+1,\left\lfloor\alpha r N^{2 / 3}\right\rfloor \rrbracket$, define horizontal increments

$$
\widetilde{I}_{i}^{z}=G_{(i-1,1), z}-G_{(i, 1), z}
$$

on the horizontal line $y=1$. Define a 2-sided walk $\left\{Z_{n}^{z, t_{0}}\right\}_{n \in \llbracket-\left\lfloor\alpha r N^{2 / 3}\right\rfloor+1,\left\lfloor\alpha r N^{2 / 3}\right\rfloor \rrbracket}$ by setting $Z_{t_{0}}^{z, t_{0}}=0$ and

$$
Z_{n}^{z, t_{0}}-Z_{n-1}^{z, t_{0}}=I_{n}-\widetilde{I}_{n}^{z}
$$

The boundary weights $I_{n}$ are those of the $\rho$-LPP process in the quadrant $-\left\lfloor r N^{2 / 3}\right\rfloor e_{1}+\mathbb{Z}_{\geq 0}^{2}$. On the event

$$
\left\{\mathbf{Z}^{-\left\lfloor r N^{2 / 3}\right\rfloor e_{1} \rightarrow z}=\left\lfloor r N^{2 / 3}\right\rfloor+t_{0}\right\}
$$

the geodesic goes through the vertical unit edge $\llbracket\left(t_{0}, 0\right),\left(t_{0}, 1\right) \rrbracket$. This implies that the walk $\left\{Z_{n}^{z, t_{0}}\right\}_{n \in \llbracket-\left\lfloor\alpha r N^{2 / 3}\right\rfloor+1,\left\lfloor\alpha r N^{2 / 3}\right\rfloor \rrbracket}$ attains its unique maximum at $n=t_{0}$. To see this, note that for $n \in \llbracket-\left\lfloor\alpha r N^{2 / 3}\right\rfloor+1,\left\lfloor\alpha r N^{2 / 3}\right\rfloor \rrbracket \backslash\left\{t_{0}\right\}$, we have almost surely

$$
\begin{aligned}
G_{-\left\lfloor r N^{2 / 3}\right\rfloor e_{1},\left(t_{0}, 0\right)}^{\rho}+G_{\left(t_{0}, 1\right), z} & >G_{-\left\lfloor r N^{2 / 3}\right\rfloor e_{1},(n, 0)}^{\rho}+G_{(n, 1), z} \\
\Longrightarrow G_{-\left\lfloor r N^{2 / 3}\right\rfloor e_{1},\left(t_{0}, 0\right)}^{\rho}-G_{-\left\lfloor r N^{2 / 3}\right\rfloor e_{1},(n, 0)}^{\rho} & >G_{(n, 1), z}-G_{\left(t_{0}, 1\right), z} .
\end{aligned}
$$




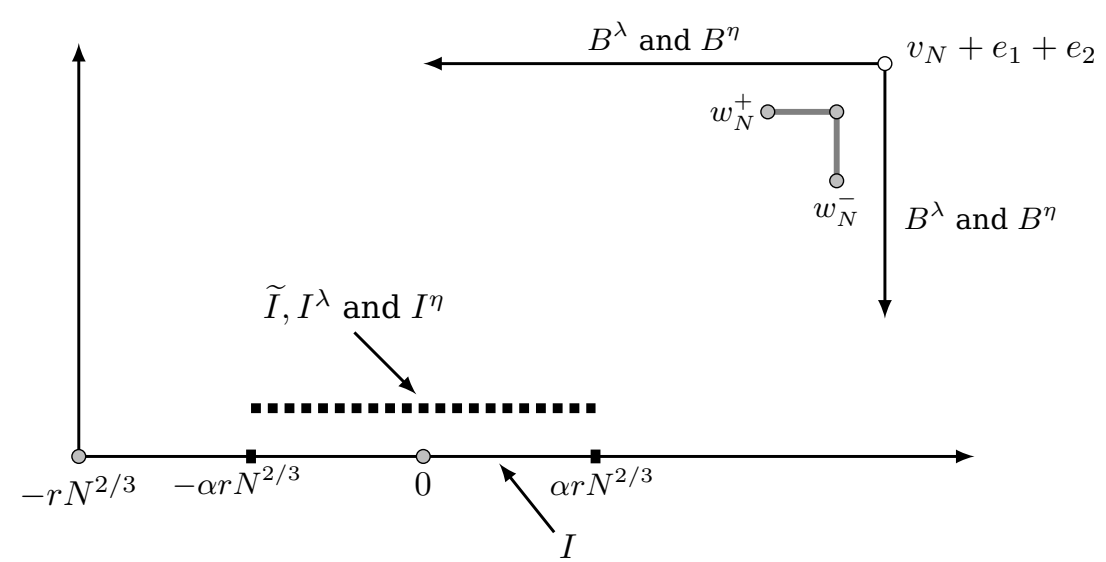

Figure 4.8: Setup for the stationary LPP processes with Busemann increments.

From this

- for $n>t_{0},(4.22) \Longrightarrow-\sum_{i=t_{0}+1}^{n} I_{i}>-\sum_{i=t_{0}+1}^{n} \widetilde{I}_{i}^{z} \Longrightarrow 0>Z_{n}^{z, t_{0}}-Z_{t_{0}}^{z, t_{0}}$;

- for $n<t_{0},(4.22) \Longrightarrow \sum_{i=n+1}^{t_{0}} I_{i}>\sum_{i=n+1}^{t_{0}} \widetilde{I}_{i}^{z} \Longrightarrow Z_{t_{0}}^{z, t_{0}}-Z_{n}^{z, t_{0}}>0$.

Since $\delta \leq \frac{1}{3} \alpha r, t_{0} \in\left[-\frac{1}{3} \alpha r N^{2 / 3}, \frac{1}{3} \alpha r N^{2 / 3}\right]$. Also because the value of the walk at $t_{0}$ is zero, we now have

$$
\begin{aligned}
\text { (4.21) } \leq & \mathbb{P}\left\{\exists z \in \mathcal{D}: \underset{n \in \mathbb{I}-\left\lfloor\alpha r N^{2 / 3}\right\rfloor+1,\left\lfloor\alpha r N^{2 / 3}\right\rfloor \mathbb{I}}{\operatorname{argmax}}\left\{Z_{n}^{z, t_{0}}\right\}=t_{0}\right\} \\
\leq & \mathbb{P}\left(\left\{\exists z \in \mathcal{D}: Z_{n}^{z, t_{0}}<0 \text { for } n \in\left(t_{0}, t_{0}+\left\lfloor\frac{1}{2} \alpha r N^{2 / 3}\right\rfloor\right]\right\}\right. \\
& \left.\bigcap\left\{\exists z \in \mathcal{D}: Z_{n}^{z, t_{0}}<0 \text { for } n \in\left[t_{0}-\left\lfloor\frac{1}{2} \alpha r N^{2 / 3}\right\rfloor, t_{0}\right)\right\}\right)
\end{aligned}
$$

Due to the relative positions of $w_{N}^{ \pm}$and $z$, Lemma 3.1 implies that

$$
\widetilde{I}_{i}^{w_{N}^{-}} \leq \widetilde{I}_{i}^{z} \leq \widetilde{I}_{i}^{w_{N}^{+}} \quad \forall i \in \llbracket-\left\lfloor\alpha r N^{2 / 3}\right\rfloor+1,\left\lfloor\alpha r N^{2 / 3}\right\rfloor \rrbracket \text { and } z \in \mathcal{D} .
$$

Hence for any $z \in \mathcal{D}$,

$$
Z_{n}^{z, t_{0}} \geq Z_{n}^{w_{N}^{+}, t_{0}} \quad \text { for } n>t_{0} \quad \text { and } \quad Z_{n}^{z, t_{0}} \geq Z_{n}^{w_{N}^{-}, t_{0}} \quad \text { for } n<t_{0} .
$$

Therefore, we may bound (4.23) by

$$
\begin{aligned}
(4.23) \leq \mathbb{P}\left(\left\{Z_{n}^{w_{N}^{+}, t_{0}}<0 \text { for } n \in\left(t_{0}, t_{0}+\left\lfloor\frac{1}{2} \alpha r N^{2 / 3}\right\rfloor\right]\right\}\right. \\
\left.\bigcap\left\{Z_{n}^{w_{N}^{-}, t_{0}}<0 \text { for } n \in\left[t_{0}-\left\lfloor\frac{1}{2} \alpha r N^{2 / 3}\right\rfloor, t_{0}\right)\right\}\right) .
\end{aligned}
$$

We bring the Busemann increments defined by the bulk weights $\left\{\omega_{x}\right\}_{x \in-\left\lfloor r N^{2 / 3}\right\rfloor e_{1}+\mathbb{Z}_{>0}^{2}}$ into the picture. To each edge on the the north and east sides of the rectangle $\llbracket-\left\lfloor r N^{2 / 3}\right\rfloor e_{1}, v_{N}+e_{1}+e_{2} \rrbracket$, we attach $\lambda$ - and $\eta$-directed Busemann increments, coupled as in Proposition 3.8. This is depicted in Figure 4.8. Together with the bulk weights in $\llbracket-\left\lfloor r N^{2 / 3}\right\rfloor e_{1}+e_{2}, v_{N} \rrbracket$, these define stationary LPP processes with north and east 
boundaries, denoted by $G_{x, v_{N}+e_{1}+e_{2}}^{\lambda, N E}$ and $G_{x, v_{N}+e_{1}+e_{2}}^{\eta, N E}$ for $x \in \llbracket\left(-\left\lfloor r N^{2 / 3}\right\rfloor, 1\right), v_{N} \rrbracket$. This is the construction explained after Theorem 3.7.

On the horizontal line $y=1$ we have for $i \in \llbracket-\left\lfloor\alpha r N^{2 / 3}\right\rfloor+1,\left\lfloor\alpha r N^{2 / 3}\right\rfloor \rrbracket$ the increments

$$
\begin{aligned}
I_{i}^{\lambda} & =G_{(i-1,1), v_{N}+e_{1}+e_{2}}^{\lambda, N E}-G_{(i, 1), v_{N}+e_{1}+e_{2}}^{\lambda, N E}=B_{(i-1,1),(i, 1)}^{\lambda} \\
\text { and } \quad I_{i}^{\eta} & =G_{(i-1,1), v_{N}+e_{1}+e_{2}}^{\eta, N E}-G_{(i, 1), v_{N}+e_{1}+e_{2}}^{\eta, N E}=B_{(i-1,1),(i, 1)}^{\eta},
\end{aligned}
$$

where the latter equalities are instances of (3.12).

Lemma 4.6. The event

$$
A=\left\{\forall i \in \llbracket-\left\lfloor\alpha r N^{2 / 3}\right\rfloor+1,\left\lfloor\alpha r N^{2 / 3}\right\rfloor \rrbracket: I_{i}^{\eta} \leq \widetilde{I}_{i}^{w_{N}^{-}} \leq \widetilde{I}_{i}^{w_{N}^{+}} \leq I_{i}^{\lambda}\right\}
$$

satisfies $\mathbb{P}\left(A^{c}\right) \leq e^{-C r^{3}}$.

Proof. The middle inequality is already in (4.24). We give the proof for

$$
\mathbb{P}\left\{\forall i \in \llbracket-\left\lfloor\alpha r N^{2 / 3}\right\rfloor+1,\left\lfloor\alpha r N^{2 / 3}\right\rfloor \rrbracket: \widetilde{I}_{i}^{w_{N}^{+}} \leq I_{i}^{\lambda}\right\} \geq 1-e^{-C r^{3}} .
$$

The similar argument for the remaining part is omitted.

We argue first that $\widetilde{I}_{i}^{w_{N}^{+}} \leq I_{i}^{\lambda}$ is implied for the entire range of indices $i$ when the geodesic of $G_{\left(\left\lfloor\alpha r N^{2 / 3}\right\rfloor, 1\right), v_{N}+e_{1}+e_{2}}^{\lambda, N E}$ exits the north boundary to the left of the point $w_{N}^{+}+e_{2}$.

For $x \in \llbracket\left(-\left\lfloor r N^{2 / 3}\right\rfloor, 1\right), w_{N}^{+}+e_{2} \rrbracket$, let $G_{x, w_{N}^{+}+e_{2}}^{\lambda, N}$ denote the last-passage time from $x$ to $w_{N}^{+}+e_{2}$ that uses the $B^{\lambda}$ increment weights on the north boundary (superscript $N$ for north).

The exit time $\mathbf{Z}^{\lambda, N E, x \rightarrow v_{N}+e_{1}+e_{2}}$ records the signed distance from the vertex $v_{N}+$ $e_{1}+e_{2}$ to the point where the geodesic of $G_{x, v_{N}+e_{1}+e_{2}}^{\lambda, N E}$ enters the north (as a positive value) or the east (as a negative value) boundary of the rectangle $\llbracket x, v_{N}+e_{1}+e_{2} \rrbracket$. Since geodesics cannot cross, the event

$$
\left\{\mathbf{Z}^{\lambda, N E,\left(\left\lfloor\alpha r N^{2 / 3}\right\rfloor, 1\right) \rightarrow v_{N}+e_{1}+e_{2}}>q r N^{2 / 3}\right\}
$$

implies

$$
\bigcap_{i \in \llbracket-\left\lfloor\alpha r N^{2 / 3}\right\rfloor+1,\left\lfloor\alpha r N^{2 / 3}\right\rfloor \rrbracket}\left\{\mathbf{Z}^{\lambda, N E,(i, 1) \rightarrow v_{N}+e_{1}+e_{2}}>q r N^{2 / 3}\right\} .
$$

This further implies

$$
\begin{aligned}
G_{(i-1,1), w_{N}^{+}+e_{2}}^{\lambda, N}-G_{(i, 1), w_{N}^{+}+e_{2}}^{\lambda, N}=G_{(i-1,1), v_{N}+e_{1}+e_{2}}^{\lambda, N E}-G_{(i, 1), v_{N}+e_{1}+e_{2}}^{\lambda, N E} & \\
& \forall i \in \llbracket-\left\lfloor\alpha r N^{2 / 3}\right\rfloor+1,\left\lfloor\alpha r N^{2 / 3}\right\rfloor \rrbracket .
\end{aligned}
$$

In the derivation below, Lemma 3.1 gives the first inequality. The equality in the second line is (4.28) which is valid on the event $\left\{\mathbf{Z}^{\lambda, N E,\left(\left\lfloor\alpha r N^{2 / 3}\right\rfloor, 1\right) \rightarrow v_{N}+e_{1}+e_{2}}>q r N^{2 / 3}\right\}$ :

$$
\begin{aligned}
& \widetilde{I}_{i}^{w_{N}^{+}}=G_{(i-1,1), w_{N}^{+}}-G_{(i, 1), w_{N}^{+}} \leq G_{(i-1,1), w_{N}^{+}+e_{2}}^{\lambda, N}-G_{(i, 1), w_{N}^{+}+e_{2}}^{\lambda, N} \\
&=G_{(i-1,1), v_{N}+e_{1}+e_{2}}^{\lambda, N E}-G_{(i, 1), v_{N}+e_{1}+e_{2}}^{\lambda, N E}=I_{i}^{\lambda} \\
& \forall i \in \llbracket-\left\lfloor\alpha r N^{2 / 3}\right\rfloor+1,\left\lfloor\alpha r N^{2 / 3}\right\rfloor \rrbracket .
\end{aligned}
$$

This finishes the proof that $\mathbf{Z}^{\lambda, N E,\left(\left\lfloor\alpha r N^{2 / 3}\right\rfloor, 1\right) \rightarrow v_{N}+e_{1}+e_{2}}>q r N^{2 / 3} \operatorname{implies} \widetilde{I}_{i}^{w_{N}^{+}} \leq I_{i}^{\lambda}$ for all $i \in \llbracket-\left\lfloor\alpha r N^{2 / 3}\right\rfloor+1,\left\lfloor\alpha r N^{2 / 3}\right\rfloor \rrbracket$. 

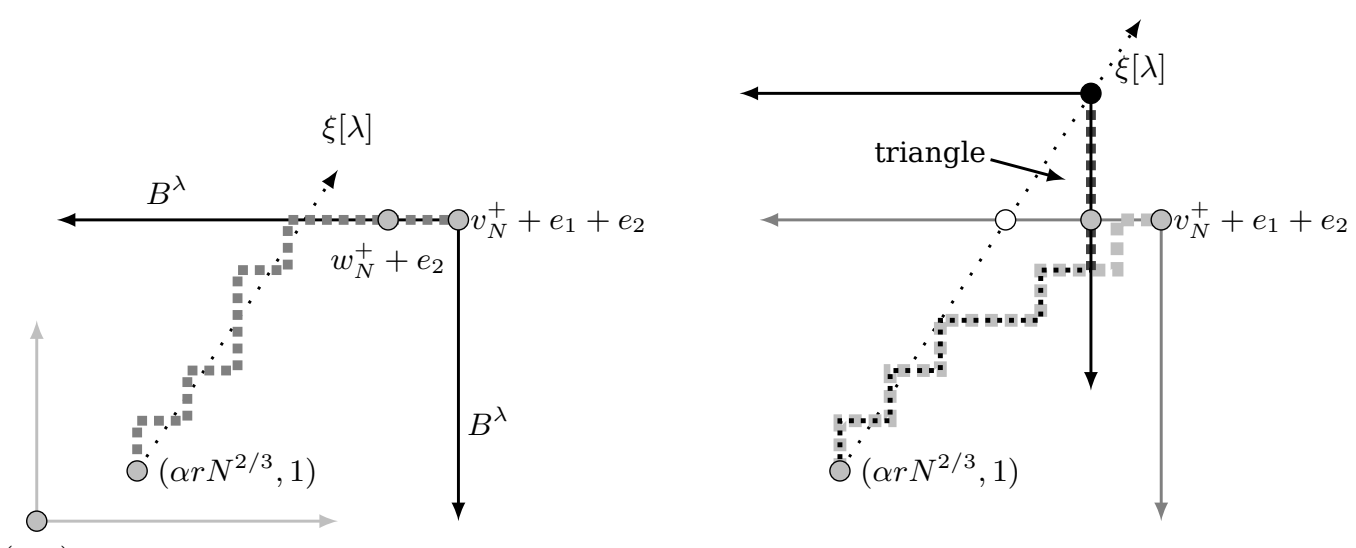

$(0,0)$

Figure 4.9: Left: The likely behavior of the geodesic of $G_{\left(\left\lfloor\alpha r N^{2 / 3}\right\rfloor, 1\right), v_{N}+e_{1}+e_{2}}^{\lambda, N E}$. It enters the north boundary to the left of $w_{N}^{+}+e_{2}$. Right: The unlikely behavior of the geodesic of $G_{\left(\left\lfloor\alpha r N^{2 / 3}\right\rfloor, 1\right), v_{N}+e_{1}+e_{2}}^{\lambda, N E}$. In this case, the dark dotted line is the geodesic between the black dot and $\left(\left\lfloor\alpha r N^{2 / 3}\right\rfloor, 1\right)$. It spends an atypically large amount of time on the boundary.

Finally, we show that

$$
\mathbb{P}\left\{\mathbf{Z}^{\lambda, N E,\left(\left\lfloor\alpha r N^{2 / 3}\right\rfloor, 1\right) \rightarrow v_{N}+e_{1}+e_{2}}>q r N^{2 / 3}\right\} \geq 1-e^{-C r^{3}}
$$

This follows from the standard exit time estimate. As shown in the left diagram of Figure 4.9, the geodesic of $G_{\left(\left\lfloor\alpha r N^{2 / 3}\right\rfloor, 1\right), v_{N}+e_{1}+e_{2}}^{\lambda, N E}$ (gray dotted line) tends to follow the characteristic direction $\xi[\lambda]$ which means it enters the north boundary on the left of $w_{N}^{+}+e_{2}$. Else, by Lemma 4.3 , there exists a parameter- $\lambda$ stationary LPP process whose geodesic (black dotted line in the right diagram of Figure 4.9) in the characteristic direction spends excessive time on the boundary. The precise argument goes as follows.

Consider the right triangle whose vertices are the black, gray and white dots highlighted in the right diagram of Figure 4.9. The distance between the white and gray dots is bounded below by $\frac{1-\rho}{2} r N^{2 / 3}$ by (4.20). Then, the distance between the black dot and the gray dot is at least $\frac{\lambda^{2}}{(1-\lambda)^{2}} \frac{1-\rho}{2} r N^{2 / 3}$ where $\frac{\lambda^{2}}{(1-\lambda)^{2}}$ is the slope of the hypotenuse. By Theorem 3.5, the probability that the geodesic shown as the black dotted line remains on the boundary throughout the segment between the black and the gray dot is bounded above by $e^{-C r^{3}}$. Here $C$ depends on $\lambda$, and bounds (4.18) turn this into a dependence on $\rho$. This completes the proof of Lemma 4.6.

With these new horizontal increments $I^{\lambda}$ and $I^{\eta}$, define two more 2-sided random walks $Z_{n}^{\lambda, t_{0}}$ and $Z_{n}^{\eta, t_{0}}$ with $Z_{t_{0}}^{\lambda, t_{0}}=Z_{t_{0}}^{\eta, t_{0}}=0$ and

$$
\begin{aligned}
& Z_{n}^{\lambda, t_{0}}-Z_{n-1}^{\lambda, t_{0}}=I_{n}-I_{n}^{\lambda}, \\
& Z_{n}^{\eta, t_{0}}-Z_{n-1}^{\eta, t_{0}}=I_{n}-I_{n}^{\eta},
\end{aligned}
$$

On the event $A$ from (4.27),

$$
Z_{n}^{\lambda, t_{0}} \leq Z_{n}^{w_{N}^{+}, t_{0}} \text { for } n>t_{0} \text { and } Z_{n}^{\eta, t_{0}} \leq Z_{n}^{w_{N}^{-}, t_{0}} \text { for } n<t_{0}
$$


We continue our bound

$$
\begin{array}{r}
\mathbb{P}(\text { event in (4.25) } \cap A) \leq \mathbb{P}\left(\left\{Z_{n}^{\lambda, t_{0}}<0 \text { for } n \in\left(t_{0}, t_{0}+\left\lfloor\frac{1}{2} \alpha r N^{2 / 3}\right\rfloor\right]\right\}\right. \\
\left.\bigcap\left\{Z_{n}^{\eta, t_{0}}<0 \text { for } n \in\left[t_{0}-\left\lfloor\frac{1}{2} \alpha r N^{2 / 3}\right\rfloor, t_{0}\right)\right\}\right) .
\end{array}
$$

From Proposition 3.8, the increment variables $\left\{I_{(i, 1)}^{\lambda}\right\}_{i>t_{0}} \cup\left\{I_{(i, 1)}^{\eta}\right\}_{i \leq t_{0}}$ are independent, and these are independent of the boundary weights $\left\{I_{i}\right\}$ by construction. Thus, the two events on the right-hand side above are independent. This gives

$$
\begin{aligned}
(4.29)=\mathbb{P}\left\{Z_{n}^{\lambda, t_{0}}<0 \text { for } n\right. & \left.\in\left(t_{0}, t_{0}+\left\lfloor\frac{1}{2} \alpha r N^{2 / 3}\right\rfloor\right]\right\} \\
\cdot & \mathbb{P}\left\{Z_{n}^{\eta, t_{0}}<0 \text { for } n \in\left[t_{0}-\left\lfloor\frac{1}{2} \alpha r N^{2 / 3}\right\rfloor, t_{0}\right)\right\} .
\end{aligned}
$$

The steps of the random walks in the two probabilities above have distributions $\operatorname{Exp}(1-$ $\rho)-\operatorname{Exp}(1-\lambda)$ and $\operatorname{Exp}(1-\eta)-\operatorname{Exp}(1-\rho)$, respectively. By Lemma A.1 each of the probabilities is bounded above by $C(\rho) r N^{-1 / 3}$ where $C(\rho)$ is a constant that depends only on $\rho$ by virtue of (4.18).

To summarize, we have shown

$$
\begin{aligned}
\mathbb{P}^{\rho} & \left\{\exists z \in \mathcal{D}: \mathbf{Z}^{-\left\lfloor r N^{2 / 3}\right\rfloor e_{1} \rightarrow z}=\left\lfloor r N^{2 / 3}\right\rfloor+t_{0}\right\} \\
& \leq \mathbb{P}\left(A^{c}\right)+\mathbb{P}^{\rho}\left(\left\{\exists z \in \mathcal{D}: \mathbf{Z}^{-\left\lfloor r N^{2 / 3}\right\rfloor e_{1} \rightarrow z}=\left\lfloor r N^{2 / 3}\right\rfloor+t_{0}\right\} \cap A\right) \\
& \leq e^{-C r^{3}}+\left(C(\rho) r N^{-1 / 3}\right)^{2} .
\end{aligned}
$$

With a union bound over $t_{0}$,

$$
\begin{aligned}
\mathbb{P}^{\rho} & \left\{\exists z \in \mathcal{D}:\left\lfloor r N^{2 / 3}\right\rfloor-\delta N^{2 / 3} \leq \mathbf{Z}^{-\left\lfloor r N^{2 / 3}\right\rfloor e_{1} \rightarrow z} \leq\left\lfloor r N^{2 / 3}\right\rfloor+\delta N^{2 / 3}\right\} \\
& \leq \mathbb{P}\left(A^{c}\right)+\mathbb{P}^{\rho}\left(\left\{\exists z \in \mathcal{D}:\left\lfloor r N^{2 / 3}\right\rfloor-\delta N^{2 / 3} \leq \mathbf{Z}^{-\left\lfloor r N^{2 / 3}\right\rfloor e_{1} \rightarrow z} \leq\left\lfloor r N^{2 / 3}\right\rfloor+\delta N^{2 / 3}\right\} \cap A\right) \\
& \leq e^{-C r^{3}}+\left(2 \delta N^{2 / 3}\right)\left(C(\rho) r N^{-1 / 3}\right)^{2} \\
& =e^{-C r^{3}}+C(\rho) 2 \delta r^{2} .
\end{aligned}
$$

Letting $r=\left(C^{-1}|\log \delta|\right)^{1 / 3}$, this gives the desired upper bound $C(\rho) \delta|\log \delta|^{2 / 3}$ with a new constant $C(\rho)$. This completes the proof for the dark region $\mathcal{D}$ of Figure 4.6.

For geodesics that enter $\mathcal{L}^{+}$we use monotonicity that comes from uniqueness of finite geodesics:

$$
\begin{gathered}
\mathbb{P}^{\rho}\left\{\exists v \in \mathcal{L}^{+}: 1 \leq \mathbf{Z}^{0 \rightarrow v} \leq \delta N^{2 / 3}\right\} \leq \mathbb{P}^{\rho}\left\{\exists v \in \mathcal{L}^{+}: \mathbf{Z}^{0 \rightarrow v} \geq 1\right\} \\
\leq \mathbb{P}^{\rho}\left\{\mathbf{Z}^{0 \rightarrow w_{N}^{+}} \geq 1\right\} \leq e^{-C r^{3}}=\delta
\end{gathered}
$$

The last inequality comes from bound (3.8) from Corollary 3.6.

For geodesics that enter $\mathcal{L}^{-}$, this follows from Lemma 4.3. First, from the uniqueness of finite geodesics, it suffices to look at the point $w_{N}^{-}$since

$$
\mathbb{P}^{\rho}\left\{\exists v \in \mathcal{L}^{-}: 1 \leq \mathbf{Z}^{0 \rightarrow v} \leq \delta N^{2 / 3}\right\} \leq \mathbb{P}^{\rho}\left\{\mathbf{Z}^{0 \rightarrow w_{N}^{-}} \leq \delta N^{2 / 3}\right\}
$$

Trace back a $(-\xi[\rho])$-directed ray from the point $w_{N}^{-}$. Up to a $\rho$-dependent constant, this ray crosses the $x$-axis at $\left\lfloor\frac{(1-\rho)^{2}}{\rho^{2}} q r N^{2 / 3}\right\rfloor e_{1}$ (the white dot in Figure 4.10). Decrease $\delta_{0}$ further if necessary so that $\delta<\delta_{0} \leq \frac{(1-\rho)^{2}}{2 \rho^{2}} q r$. Then the distance between the black and white dots in Figure 4.10 is at least $\frac{(1-\rho)^{2}}{2 \rho^{2}} q r N^{2 / 3}$.

Let $h$ be the positive integer such that $\left(\left\lfloor\delta r N^{2 / 3}\right\rfloor,-h\right)$ is the closest lattice point to the $(-\xi[\rho])$-directed ray from $w_{N}^{-}$. Then, $h \geq \frac{1}{2} q r N^{2 / 3}$. From Lemma 3.1, whenever $\mathbf{Z}^{0 \rightarrow w_{N}^{-}} \leq$ $\delta N^{2 / 3}$ (gray dotted line), then $\mathbf{Z}^{\left(\left\lfloor\delta N^{2 / 3}\right\rfloor,-h\right) \rightarrow w_{N}^{-}}<-h$ (black dotted line). Theorem 3.5 bounds this probability by $e^{-C r^{3}}$. This completes the proof of Theorem 4.5. 


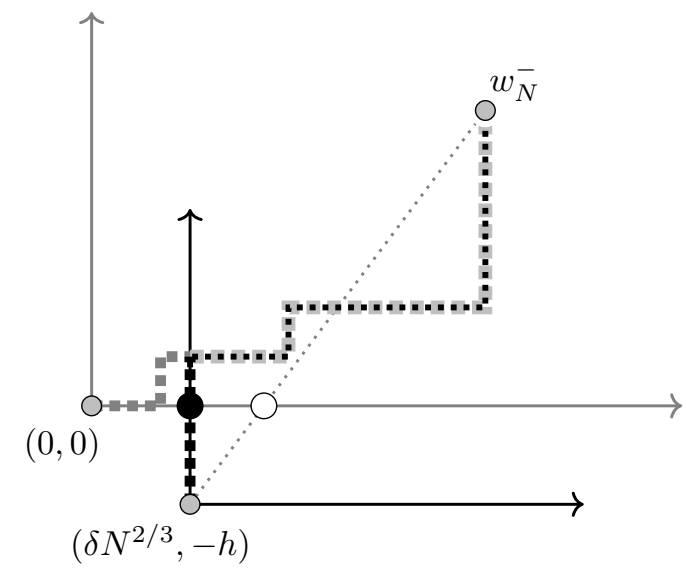

Figure 4.10: From Lemma 4.3, if $\mathbf{Z}^{0 \rightarrow w_{N}^{-}} \leq \delta N^{2 / 3}$ (gray dotted line),

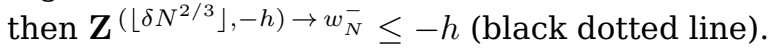

\section{Dual geodesics and proofs of the main theorems}

The main theorems from Section 2 are proved by applying the exit time bounds of Section 4 to dual geodesics that live on the dual lattice. First define south and west directed semi-infinite paths (superscript sw) in terms of the Busemann functions from Theorem 3.7:

$$
\begin{aligned}
& \mathbf{b}_{0}^{\mathrm{sw}, \rho, x}=x, \quad \text { and for } k \geq 0
\end{aligned}
$$

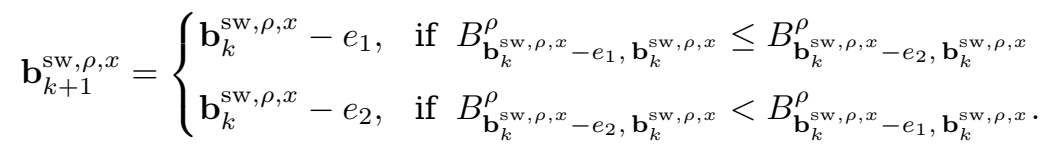

Recall the dual weights $\left\{\breve{\omega}_{x}^{\rho}=B_{x-e_{1}, x}^{\rho} \wedge B_{x-e_{2}, x}^{\rho}\right\}_{x \in \mathbb{Z}^{2}}$ introduced in part (iii) of Theorem 3.7.

Let $e^{*}=\frac{1}{2}\left(e_{1}+e_{2}\right)=\left(\frac{1}{2}, \frac{1}{2}\right)$ denote the shift between the lattice $\mathbb{Z}^{2}$ and its dual $\mathbb{Z}^{2 *}=\mathbb{Z}^{2}+e^{*}$. Shift the dual weights to the dual lattice by defining $\omega_{z}^{*}=\check{\omega}_{z+e^{*}}^{\rho}$ for $z \in \mathbb{Z}^{2 *}$. By Theorem 3.7(iii) these weights are i.i.d. $\operatorname{Exp}(1)$. The LPP process for these weights is defined as in (2.1):

$$
G_{x, y}^{*}=\max _{z . \in \Pi^{x, y}} \sum_{k=0}^{|y-x|_{1}} \omega_{z_{k}}^{*} .
$$

Shift the southwest paths to the dual lattice by defining

$$
\mathbf{b}_{k}^{*, \rho, z}=\mathbf{b}_{k}^{\mathrm{sw}, \rho, z+e^{*}}-e^{*} \quad \text { for } z \in \mathbb{Z}^{2 *} \text { and } k \geq 0 .
$$

These definitions reproduce on the dual lattice the semi-infinite geodesic setting described in Section 3.3, with reflected lattice directions. This is captured in the next theorem that summarizes the development from Section 4.2 of [28].

Theorem 5.1. Fix $\rho \in(0,1)$. Then the following hold almost surely.

(i) For each $z \in \mathbb{Z}^{2 *}$, the path $\mathbf{b}^{*, \rho, z}$ is the unique $(-\xi[\rho])$-directed semi-infinite geodesic from $z$ in the LPP process (5.2). Precisely,

$$
\lim _{n \rightarrow \infty} \frac{\mathbf{b}_{n}^{*, \rho, z}}{n}=-\xi[\rho] \text { and } \forall k<l \text { in } \mathbb{Z}_{\geq 0}: G_{\mathbf{b}_{l}^{*, \rho, z}, \mathbf{b}_{k}^{*, \rho, z}}^{*}=\sum_{i=k}^{l} \omega_{\mathbf{b}_{i}^{*, \rho, z}}^{*}
$$




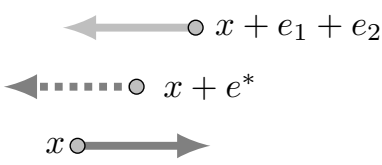

Figure 5.1: The equivalent events $\mathbf{b}_{1}^{\rho, x}=x+e_{1}$ (dark gray arrow), $\mathbf{b}_{1}^{\mathrm{sw}, \rho, x+e_{1}+e_{2}}=x+e_{2}$ (light gray arrow), and $\mathbf{b}_{k}^{*, \rho, x+e^{*}}=x+e^{*}-e_{1}$ (dotted arrow). The dark gray and dotted arrows never cross.

(ii) The semi-infinite geodesics and the dual semi-infinite geodesics are equal in distribution, modulo the $e^{*}$-shift and lattice reflection: $\left\{\mathbf{b}^{*, \rho, z}\right\}_{z \in \mathbb{Z}^{* 2}} \stackrel{d}{=}\left\{-e^{*}-\right.$ $\left.\mathbf{b}^{\rho,-\left(z+e^{*}\right)}\right\}_{z \in \mathbb{Z}^{* 2}}$.

(iii) The collections of paths $\left\{\mathbf{b}^{\rho, z}\right\}_{z \in \mathbb{Z}^{2}}$ and $\left\{\mathbf{b}^{*, \rho, z}\right\}_{z \in \mathbb{Z}^{* 2}}$ almost surely never cross each other.

Part (ii), the distributional equality of the tree of directed geodesics and the dual, was first proved in [24]. The non-crossing property of part (iii) can be seen from a simple picture. The additivity of the Busemann functions gives

$$
B_{x, x+e_{1}}^{\rho}+B_{x+e_{1}, x+e_{1}+e_{2}}^{\rho}=B_{x, x+e_{2}}^{\rho}+B_{x+e_{2}, x+e_{1}+e_{2}}^{\rho} .
$$

By (3.9) $\mathbf{b}_{1}^{\rho, x}=x+e_{1}$ if and only if $B_{x, x+e_{1}}^{\rho} \leq B_{x, x+e_{2}}^{\rho}$. By (5.3) this is equivalent to $B_{x+e_{2}, x+e_{1}+e_{2}}^{\rho} \leq B_{x+e_{1}, x+e_{1}+e_{2}}^{\rho}$ which is the same as $\mathbf{b}_{1}^{\mathrm{sw}, \rho, x+e_{1}+e_{2}}=x+e_{2}$, and this last is equivalent to $\mathbf{b}_{k}^{*, \rho, x+e^{*}}=x+e^{*}-e_{1}$. An analogous argument works for the $e_{2}$ step. The conclusion is that the increments of $\mathbf{b}^{\rho, \bullet}$ out of $x$ and $\mathbf{b}^{*, \rho, \bullet}$ out of $x+e^{*}$ cannot cross. See Figure 5.1.

To connect the dual semi-infinite geodesics with $\rho$-geodesics, define a stationary

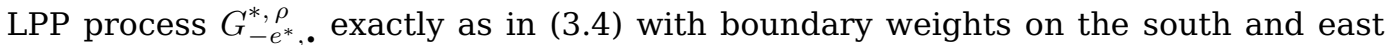
boundaries, but on the dual quadrant $-e^{*}+\mathbb{Z}_{>0}^{2}$ based at $-e^{*}$. The boundary weights are defined by shifting Busemann function values to the dual lattice:

$$
I_{-e^{*}+k e_{1}}^{*, \rho}=B_{(k-1) e_{1}, k e_{1}}^{\rho} \quad \text { and } \quad J_{-e^{*}+l e_{2}}^{*, \rho}=B_{(l-1) e_{1}, l e_{1}}^{\rho} .
$$

The bulk weights are $\left\{\omega_{x}^{*}: x \in \mathbb{Z}^{* 2}, x \geq e^{*}\right\}$.

Proposition 5.2. For any $w \in e^{*}+\mathbb{Z}_{\geq 0}^{2}$ the following holds. The edges of the semiinfinite geodesic $\mathbf{b}^{*, \rho, w}$ that have at least one endpoint in $e^{*}+\mathbb{Z}_{\geq_{0}}^{2}$ are also edges of the geodesic of $G_{-e^{*}, w}^{*}$.

Proposition 5.2, illustrated in Figure 5.2, is another version of Lemma 3.2. It is proved as Prop. 5.1 in [28] but without the shift to the dual lattice, so in terms of the southwest geodesics in (5.1) for the weights $\check{\omega}^{\rho}$.

We are ready to prove the main results.

Proof of Theorem 2.2. Referring to Figure 5.3, geodesics $\mathbf{b}^{\rho,\left(0,\left\lfloor\delta N^{2 / 3}\right\rfloor\right)}$ and $\mathbf{b}^{\rho,\left(\left\lfloor\delta N^{2 / 3}\right\rfloor, 0\right)}$ (gray dotted lines) coalesce outside $\llbracket 0, v_{N} \rrbracket$ if and only if some dual geodesic started outside of $\llbracket 0, v_{N} \rrbracket-e^{*}$ (black dotted line) enters the square $\llbracket(0,0),\left(\left\lfloor\delta N^{2 / 3}\right\rfloor,\left\lfloor\delta N^{2 / 3}\right\rfloor\right) \rrbracket$. From Proposition 5.2, the restrictions of these dual geodesics are the $\rho$-geodesics of the stationary LPP process on $-e^{*}+\mathbb{Z}_{\geq 0}^{2}$ with Busemann boundary weights on the south and west. Consequently

$$
\mathbb{P}\left\{\mathbf{z}^{\rho}\left(\left\lfloor\delta N^{2 / 3}\right\rfloor e_{1},\left\lfloor\delta N^{2 / 3}\right\rfloor e_{2}\right) \notin \llbracket 0, v_{N} \rrbracket\right\}=\mathbb{P}^{\rho}\left\{\exists z \notin \llbracket 0, v_{N} \rrbracket:\left|\mathbf{Z}^{0 \rightarrow z}\right| \leq \delta N^{2 / 3}\right\} .
$$

The bounds claimed in Theorem 2.2 follow from Theorems 4.4 and 4.5. 

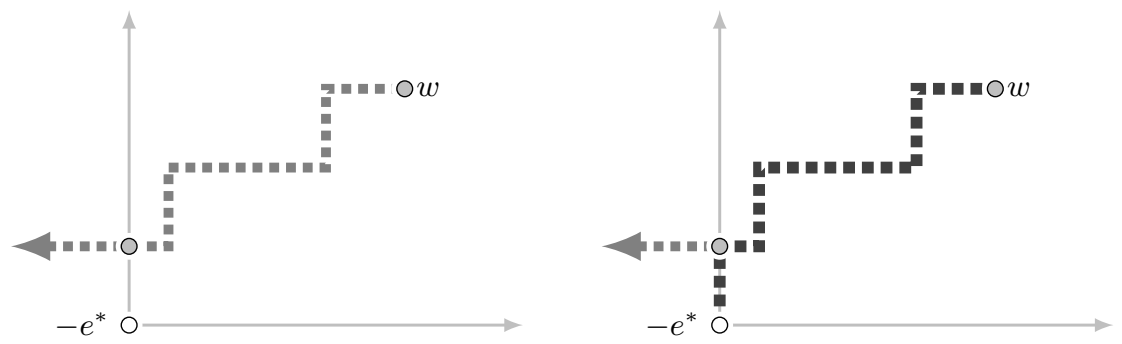

Figure 5.2: Illustration of Proposition 5.2. On the left the dual semiinfinite geodesic $\mathbf{b}^{*, \rho, w}$ (light dotted path). On the right the geodesic of $G_{-e^{*}, w}^{*, \rho}$ (dark dotted path). The two paths coincide in the bulk.

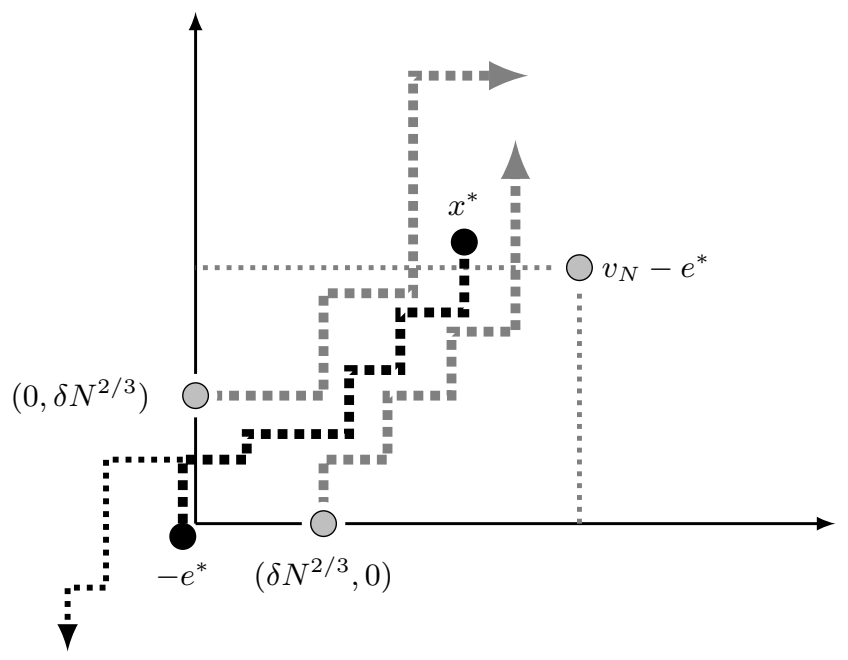

Figure 5.3: Geodesics $\mathbf{b}^{\rho,\left(\left\lfloor\delta N^{2 / 3}\right\rfloor, 0\right)}$ and $\mathbf{b}^{\rho,\left(0,\left\lfloor\delta N^{2 / 3}\right\rfloor\right)}$ (gray dotted lines) coalesce outside $\llbracket 0, v_{N} \rrbracket$. Equivalently, some dual point $x^{*}$ outside of $\llbracket 0, v_{N} \rrbracket-e^{*}$ sends a dual geodesic (black dotted line) into the rectangle $\llbracket(0,0),\left(\left\lfloor\delta N^{2 / 3}\right\rfloor,\left\lfloor\delta N^{2 / 3}\right\rfloor\right) \rrbracket$.

Proof of Theorem 2.3. Referring to Figure 5.4, geodesics $\mathbf{b}^{\rho,\left(0,\left\lfloor r N^{2 / 3}\right\rfloor\right)}$ and $\mathbf{b}^{\rho,\left(\left\lfloor r N^{2 / 3}\right\rfloor, 0\right)}$ (gray dotted lines) coalesce inside $\llbracket 0, v_{N} \rrbracket$ if and only if every dual geodesic started from the north and east boundaries of $\llbracket-e^{*}, v_{N}+e^{*} \rrbracket$ (black dotted lines) avoids the square $\llbracket(0,0),\left(\left\lfloor r N^{2 / 3}\right\rfloor,\left\lfloor r N^{2 / 3}\right\rfloor\right) \rrbracket$. From Proposition 5.2, the restrictions of these dual geodesics are the $\rho$-geodesics of the stationary LPP process on $-e^{*}+\mathbb{Z}_{\geq 0}^{2}$ with Busemann boundary weights on the south and west,

$$
\mathbb{P}\left\{\mathbf{z}^{\rho}\left(\left\lfloor r N^{2 / 3}\right\rfloor e_{1},\left\lfloor r N^{2 / 3}\right\rfloor e_{2}\right) \in \llbracket 0, v_{N} \rrbracket\right\}=\mathbb{P}^{\rho}\left\{\forall z \notin \llbracket 0, v_{N} \rrbracket:\left|\mathbf{Z}^{0 \rightarrow z}\right| \geq r N^{2 / 3}\right\} .
$$

The lower bound claimed in Theorem 2.3 follows from Theorem 4.1. The claimed upper bound is a trivial weakening of Theorem 3.5.

Proof of Corollary 2.4. From the duality, it suffices to show

(i) $\mathbb{P}^{\rho}\left\{\exists z\right.$ outside $\llbracket 0, v_{N} \rrbracket$ such that $\left.1 \leq \mathbf{Z}^{0 \rightarrow z} \leq \delta N^{2 / 3}\right\} \geq C_{1} \delta$;

(ii) $\mathbb{P}^{\rho}\left\{\exists z\right.$ outside $\llbracket 0, v_{N} \rrbracket$ such that $\left.1 \leq \mathbf{Z}^{0 \rightarrow z} \leq r N^{2 / 3}\right\} \geq 1-e^{-C_{2} r^{3}}$. 


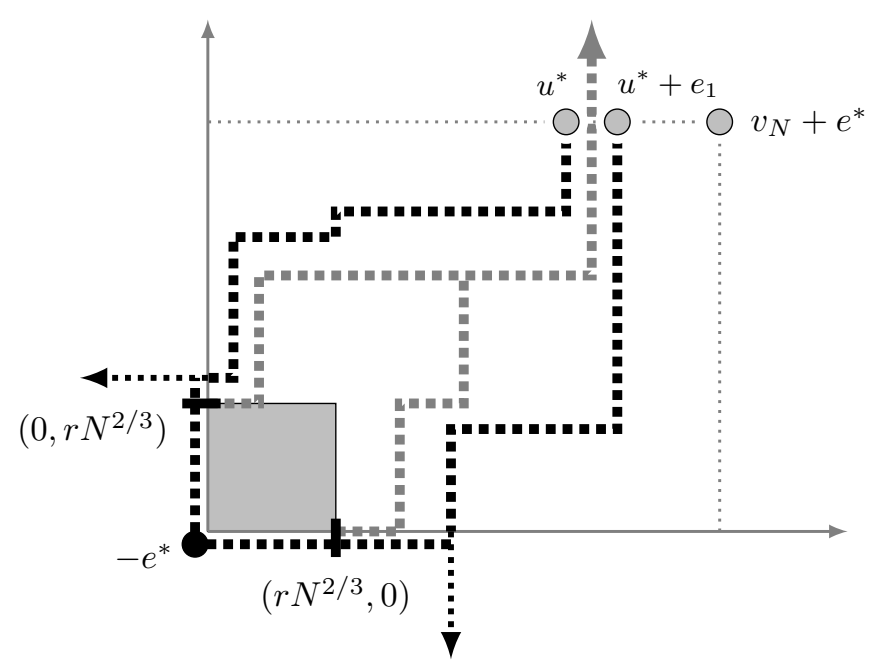

Figure 5.4: None of the the $\rho$-geodesics will enter the gray square because they are bounded away by the two dual geodesics (black dotted lines) drawn above.

We establish (ii) from the special case

$$
\mathbb{P}^{\rho}\left\{1 \leq \mathbf{Z}^{0 \rightarrow v_{N}+\left\lfloor\frac{1}{10} r N^{2 / 3}\right\rfloor e_{1}} \leq r N^{2 / 3}\right\} \geq 1-e^{-C_{2} r^{2}}
$$

Furthermore, from (5.6) the proof of Theorem 4.4 can be adapted to prove (i), by partitioning $\left[0, r N^{2 / 3}\right]$ into intervals of size $\leq \delta r N^{2 / 3}$ and repeating the argument.

Inequality (5.6) comes from the estimates

$$
\begin{aligned}
& \mathbb{P}^{\rho}\left\{\mathbf{Z}^{0 \rightarrow v_{N}+\left\lfloor\frac{1}{10} r N^{2 / 3}\right\rfloor e_{1}} \leq-1\right\} \leq e^{-C r^{3}} \\
& \mathbb{P}^{\rho}\left\{\mathbf{Z}^{0 \rightarrow v_{N}+\left\lfloor\frac{1}{10} r N^{2 / 3}\right\rfloor e_{1}}>r N^{2 / 3}\right\} \leq e^{-C r^{3}} .
\end{aligned}
$$

Inequality (5.7) is bound (3.7) of Corollary 3.6. For (5.8), apply Lemma 3.4 to the process $G_{z, \bullet}^{(0), \rho}$ with the new base point $z=\left\lfloor\frac{1}{10} r N^{2 / 3}\right\rfloor e_{1}$, and then Theorem 3.5:

$$
\mathbb{P}^{\rho}\left\{\mathbf{Z}^{0 \rightarrow v_{N}+\left\lfloor\frac{1}{10} r N^{2 / 3}\right\rfloor e_{1}} \geq r N^{2 / 3}\right\} \leq \mathbb{P}^{\rho}\left\{\mathbf{Z}^{0 \rightarrow v_{N}} \geq \frac{9}{10} r N^{2 / 3}\right\} \leq e^{-C r^{3}} .
$$

Proof of Theorem 2.8. If the semi-infinite geodesic $\mathbf{b}^{\rho,(0,0)}$ enters the interior of the square $\llbracket v_{N}-\left(\delta N^{2 / 3}, \delta N^{2 / 3}\right), v_{N} \rrbracket$ as shown in Figure 5.5, we obtain a $\rho$-geodesic from Proposition 5.2 whose exit time satisfies $\left|\mathbf{Z}^{N E, 0 \rightarrow v_{N}}\right| \leq \delta N^{2 / 3}$. Applying the exit time estimate Theorem 4.5 finishes the proof.

\section{A Appendix}

Below is the random walk estimate for the proof of Theorem 4.5. It is proved as Lemma C.1 in Appendix C of [2].

Lemma A.1. Let $\alpha>\beta>0$. Let $S_{n}=\sum_{k=1}^{n} Z_{k}$ be a random walk with step distribution $Z_{k} \sim \operatorname{Exp}(\alpha)-\operatorname{Exp}(\beta)$ (difference of independent exponentials). Then there is an absolute constant $C$ independent of all the parameters such that for $n \in \mathbb{Z}_{>0}$,

$$
\mathbb{P}\left(S_{1}<0, S_{2}<0, \cdots, S_{n}<0\right) \leq \frac{C}{\sqrt{n}}\left(1-\frac{(\alpha-\beta)^{2}}{(\alpha+\beta)^{2}}\right)^{n}+\frac{\alpha-\beta}{\alpha}
$$




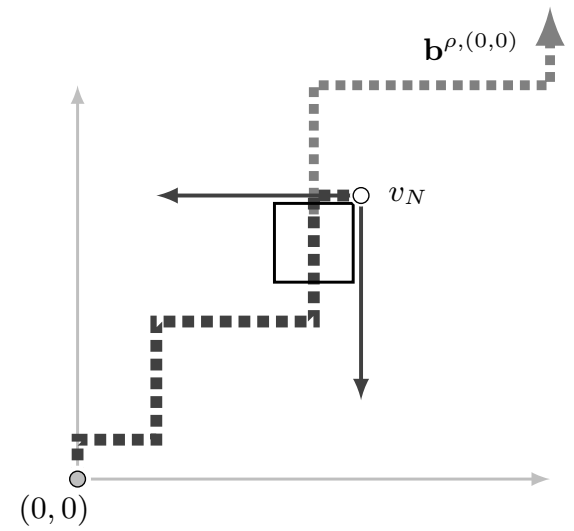

Figure 5.5: The square in the picture is $\llbracket v_{N}-\left(\delta N^{2 / 3}, \delta N^{2 / 3}\right), v_{N} \rrbracket$. We obtain a $\rho$-geodesic with north and east boundaries from the semiinfinite geodesic in gray.

Next the moment bound on the Radon-Nikodym for the proof of Theorem 4.1.

Lemma A.2. Let $a>0, b \in \mathbb{R}$, and $N \in \mathbb{Z}_{>0}$. For $\rho>0$, let $Q^{\rho}$ be the probability distribution on the product space $\Omega=\mathbb{R}^{\left\lfloor a N^{1 / 3}\right\rfloor}$ under which the coordinates $X_{i}(\omega)=\omega_{i}$ are i.i.d. $\operatorname{Exp}(\rho)$ random variables. Assume that

$$
N \geq|b|^{3} \rho^{-3}(1-\eta)^{-3}
$$

for some $\eta \in(0,1)$. Let $f$ denote the Radon-Nikodym derivative

$$
f(\omega)=\frac{d Q^{\rho+b N^{-1 / 3}}}{d Q^{\rho}}(\omega) .
$$

Then

$$
E^{Q^{\rho}}\left[f^{2}\right] \leq \exp \left\{\frac{a b^{2}}{\rho^{2}}+\frac{10 a|b|^{3}}{3 \rho^{3} \eta N^{1 / 3}}\right\} .
$$

Proof. Let $\lambda=\rho+b N^{-1 / 3}$. Assumption (A.2) implies that $|\lambda-\rho| \leq(1-\eta) \rho$ so in particular the distribution $\operatorname{Exp}(\lambda)$ is well-defined. Note the inequality

$$
\left|\log (1+x)-x+\frac{x^{2}}{2}\right| \leq \sum_{k=3}^{\infty} \frac{|x|^{k}}{k} \leq \frac{|x|^{3}}{3 \eta}
$$

valid for $\eta \in(0,1)$ and $|x| \leq 1-\eta$. Apply it below to $x=b \rho^{-1} N^{-1 / 3}$ and $x=2 b \rho^{-1} N^{-1 / 3}$.

$$
\begin{aligned}
E^{Q^{\rho}}\left[f^{2}\right] & =\int_{\Omega}\left(\prod_{i=1}^{\left\lfloor a N^{2 / 3}\right\rfloor} \frac{\lambda e^{-\lambda \omega_{i}}}{\rho e^{-\rho \omega_{i}}}\right)^{2} Q(d \omega)=\left(\frac{\lambda^{2}}{\rho^{2}} \int_{0}^{\infty} e^{-2(\lambda-\rho) x} \rho e^{-\rho x} d x\right)^{\left\lfloor a N^{2 / 3}\right\rfloor} \\
& =\left(\frac{\lambda^{2}}{\rho(2 \lambda-\rho)}\right)^{\left\lfloor a N^{2 / 3}\right\rfloor}=\exp \left\{\left\lfloor a N^{2 / 3}\right\rfloor[2 \log \lambda-\log \rho-\log (2 \lambda-\rho)]\right\} \\
& =\exp \left\{\left\lfloor a N^{2 / 3}\right\rfloor\left[2 \log \left(1+b \rho^{-1} N^{-1 / 3}\right)-\log \left(1+2 b \rho^{-1} N^{-1 / 3}\right)\right]\right\} \\
& \leq \exp \left\{\frac{a b^{2}}{\rho^{2}}+\frac{10 a|b|^{3}}{3 \rho^{3} N^{1 / 3}}\right\} .
\end{aligned}
$$




\section{References}

[1] Gideon Amir, Omer Angel, and Benedek Valkó, The TASEP speed process, Ann. Probab. 39 (2011), no. 4, 1205-1242. MR-2857238

[2] Márton Balázs, Ofer Busani, and Timo Seppäläinen, Non-existence of bi-infinite geodesics in the exponential corner growth model, 2019, arXiv:1909.06883. MR-4116707

[3] Márton Balázs, Ofer Busani, and Timo Seppäläinen, Local stationarity of exponential last passage percolation, 2020, arXiv:1909.06883.

[4] Márton Balázs, Eric Cator, and Timo Seppäläinen, Cube root fluctuations for the corner growth model associated to the exclusion process, Electron. J. Probab. 11 (2006), no. 42, 1094-1132 (electronic). MR-2268539

[5] Márton Balázs, Júlia Komjáthy, and Timo Seppäläinen, Microscopic concavity and fluctuation bounds in a class of deposition processes, Ann. Inst. Henri Poincaré Probab. Stat. 48 (2012), no. 1, 151-187. MR-2919202

[6] Márton Balázs and Timo Seppäläinen, Order of current variance and diffusivity in the asymmetric simple exclusion process, Ann. of Math. (2) 171 (2010), no. 2, 1237-1265. MR-2630064

[7] Riddhipratim Basu, Sourav Sarkar, and Allan Sly, Coalescence of geodesics in exactly solvable models of last passage percolation, J. Math. Phys. 60 (2019), no. 9, 093301, 22. MR-4002528

[8] Manan Bhatia, Moderate deviation and exit time estimates for stationary last passage percolation, 2020, arXiv:2004.12987.

[9] Eric Cator and Piet Groeneboom, Second class particles and cube root asymptotics for Hammersley's process, Ann. Probab. 34 (2006), no. 4, 1273-1295. MR-2257647

[10] Hans Chaumont and Christian Noack, Characterizing stationary $1+1$ dimensional lattice polymer models, Electron. J. Probab. 23 (2018), Paper No. 38, 19. MR-3806406

[11] Ivan Corwin, The Kardar-Parisi-Zhang equation and universality class, Random Matrices Theory Appl. 1 (2012), no. 1, 1130001, 76. MR-2930377

[12] David Coupier, Multiple geodesics with the same direction, Electron. Commun. Probab. 16 (2011), 517-527. MR-2836758

[13] Murray Eden, A two-dimensional growth process, Proc. 4th Berkeley Sympos. Math. Statist. and Prob., Vol. IV, Univ. California Press, Berkeley, Calif., 1961, pp. 223-239. MR-0136460

[14] Elnur Emrah, Chris Janjigian, and Timo Seppäläinen, Right-tail moderate deviations in the exponential last-passage percolation, 2020, arXiv:2004.04285.

[15] Wai-Tong Louis Fan and Timo Seppäläinen, Joint distribution of Busemann functions in the exactly solvable corner growth model, 2018, arXiv:1808.09069.

[16] Pablo A. Ferrari and Leandro P. R. Pimentel, Competition interfaces and second class particles, Ann. Probab. 33 (2005), no. 4, 1235-1254. MR-2150188

[17] Nicos Georgiou, Firas Rassoul-Agha, and Timo Seppäläinen, Stationary cocycles and Busemann functions for the corner growth model, Probab. Theory Related Fields 169 (2017), no. 1-2, 177-222. MR-3704768

[18] J. M. Hammersley and D. J. A. Welsh, First-passage percolation, subadditive processes, stochastic networks, and generalized renewal theory, Proc. Internat. Res. Semin., Statist. Lab., Univ. California, Berkeley, Calif, Springer-Verlag, New York, 1965, pp. 61-110. MR0198576

[19] C. Douglas Howard and Charles M. Newman, Euclidean models of first-passage percolation, Probab. Theory Related Fields 108 (1997), no. 2, 153-170. MR-1452554

[20] C. Douglas Howard and Charles M. Newman, Geodesics and spanning trees for Euclidean first-passage percolation, Ann. Probab. 29 (2001), no. 2, 577-623. MR-1849171

[21] Christopher Janjigian, Firas Rassoul-Agha, and Timo Seppäläinen, Geometry of geodesics through Busemann measures in directed last-passage percolation, 2019, arXiv:1908.09040. MR-3838897

[22] Cristina Licea and Charles M. Newman, Geodesics in two-dimensional first-passage percolation, Ann. Probab. 24 (1996), no. 1, 399-410. MR-1387641 


\section{Coalescence estimates for the CGM}

[23] Charles M. Newman, A surface view of first-passage percolation, Proceedings of the International Congress of Mathematicians, Vol. 1, 2 (Zürich, 1994), Birkhäuser, Basel, 1995, pp. 1017-1023. MR-1404001

[24] Leandro P. R. Pimentel, Duality between coalescence times and exit points in last-passage percolation models, Ann. Probab. 44 (2016), no. 5, 3187-3206. MR-3551194

[25] Jeremy Quastel, Introduction to KPZ, Current developments in mathematics, 2011, Int. Press, Somerville, MA, 2012, pp. 125-194. MR-3098078

[26] Timo Seppäläinen, Scaling for a one-dimensional directed polymer with boundary conditions, Ann. Probab. 40 (2012), no. 1, 19-73, Corrected version available at arXiv:0911.2446. MR2917766

[27] Timo Seppäläinen, The corner growth model with exponential weights, Random growth models, Proc. Sympos. Appl. Math., vol. 75, Amer. Math. Soc., Providence, RI, 2018, arXiv:1709.05771, pp. 133-201. MR-3838898

[28] Timo Seppäläinen, Existence, uniqueness and coalescence of directed planar geodesics: proof via the increment-stationary growth process, 2018, To appear in Ann. Inst. Henri Poincaré Probab. Stat., arXiv:1812.02689. MR-4116707

[29] Mario V. Wüthrich, Asymptotic behaviour of semi-infinite geodesics for maximal increasing subsequences in the plane, In and out of equilibrium (Mambucaba, 2000), Progr. Probab., vol. 51, Birkhäuser Boston, Boston, MA, 2002, pp. 205-226. MR-1901954

Acknowledgments. The authors gratefully thank the anonymous referee for his/her suggestions about improving the exposition of this paper. 


\section{Electronic Journal of Probability Electronic Communications in Probability}

\section{Advantages of publishing in EJP-ECP}

- Very high standards

- Free for authors, free for readers

- Quick publication (no backlog)

- Secure publication $\left(\mathrm{LOCKSS}^{1}\right)$

- Easy interface (EJMS²)

\section{Economical model of EJP-ECP}

- Non profit, sponsored by $\mathrm{IMS}^{3}, \mathrm{BS}^{4}$, ProjectEuclid ${ }^{5}$

- Purely electronic

\section{Help keep the journal free and vigorous}

- Donate to the IMS open access fund ${ }^{6}$ (click here to donate!)

- Submit your best articles to EJP-ECP

- Choose EJP-ECP over for-profit journals

\footnotetext{
${ }^{1}$ LOCKSS: Lots of Copies Keep Stuff Safe http://www. lockss.org/

${ }^{2}$ EJMS: Electronic Journal Management System http://www.vtex.lt/en/ejms.html

${ }^{3}$ IMS: Institute of Mathematical Statistics http://www.imstat.org/

${ }^{4}$ BS: Bernoulli Society http://www. bernoulli-society.org/

${ }^{5}$ Project Euclid: https://projecteuclid.org/

${ }^{6}$ IMS Open Access Fund: http://www.imstat.org/publications/open.htm
} 